\title{
Premetric teleparallel theory of gravity and its local and linear constitutive law
}

\author{
Yakov Itin ${ }^{1, \mathrm{a}}$, Yuri N. Obukhov ${ }^{2, \mathrm{~b}}$, Jens Boos ${ }^{3, \mathrm{c}}$, Friedrich W. Hehl ${ }^{4, \mathrm{~d}}$ \\ ${ }^{1}$ Institute of Mathematics, The Hebrew University of Jerusalem and Jerusalem College of Technology, 91160 Jerusalem, Israel \\ 2 Theoretical Physics Laboratory, Nuclear Safety Institute, Russian Academy of Sciences, B. Tulskaya 52, 115191 Moscow, Russia \\ 3 Theoretical Physics Institute, University of Alberta, Edmonton, AB T6G 2E1, Canada \\ ${ }^{4}$ Institute for Theoretical Physics, University of Cologne, 50923 Cologne, Germany
}

Received: 23 August 2018 / Accepted: 13 October 2018 / Published online: 9 November 2018

(C) The Author(s) 2018

\begin{abstract}
We continue to investigate the premetric teleparallel theory of gravity (TG) with the coframe (tetrad) as gravitational potential. We start from the field equations and a local and linear constitutive law. We create a Tonti diagram of TG in order to disclose the structure of TG. Subsequently we irreducibly decompose the 6th order constitutive tensor under the linear group. Moreover, we construct the most general constitutive tensors from the metric and the totally antisymmetric Levi-Civita symbol, and we demonstrate that they encompass nontrivial axion and skewon type pieces. Using these tools, we derive for TG in the geometric-optics approximation propagating massless spin 0,1 , and 2 waves, including the special case of Einstein's general relativity.
\end{abstract}

\section{Contents}

1 Introduction . . . . . . . . . . . . 1

1.1 Field equations . . . . . . . . . . . . 2

1.2 Local and linear constitutive law . . . . . . . 3

1.3 Reversibility ................ 4

2 Tonti diagram of the premetric teleparallel theory of gravity ................. 5

3 Decompositions of the constitutive tensor . . . . . 6

3.1 Two forms of the constitutive tensor . . . . 6

$3.2 S_{4} \times S_{2}$ decomposition of $\chi^{\alpha \beta}{ }_{\mu}{ }^{\gamma \delta}{ }_{v} \ldots \ldots .7$

$3.3 S_{6}$ decomposition of $\check{\chi}_{\alpha \beta \mu \gamma \delta \nu} \ldots \ldots \ldots . . . \quad 8$

3.4 Relation between the decompositions . . . . . 9

3.5 Reversibility . . . . . . . . . . . . . . 9 9

3.6 Lagrangian . . . . . . . . . . . . . . . 10

a e-mail: itin@math.huji.ac.il

b e-mail: obukhov@ibrae.ac.ru

ce-mail: boos@ualberta.ca

de-mail: hehl@thp.uni-koeln.de
3.7 Parametrization of irreducible parts . . . . . . . 10

3.8 Energy-momentum current . . . . . . . . . . 10

3.9 Contracting $\chi^{\alpha \beta} \gamma_{\rho}^{\mu \nu}$ twice: four second order tensors . . . . . . . . . . . 10

4 Metric-dependent constitutive tensor . . . . . . 12

4.1 A metric-dependent ansatz with parity conserving terms ............ 12

4.2 Parity violating terms . . . . . . . . . . 13

4.3 The general case and its irreducible decomposition . . . . . . . . . . . 14

4.4 Specific Lagrangians. . . . . . . . . . . . . 14 4.4.1 $\mathrm{GR}_{\|}$: the teleparallel equivalent of GR . . 14 4.4.2 Von der Heyde Lagrangian . . . . . . . 15 4.4.3 Torsion-square Lagrangian in nonlocal gravity (NLG) . . . . . . . . . . . . 15

5 Propagation of gravitational waves . . . . . . . . 15

5.1 Geometric optics approximation . . . . . . 15

5.2 Dispersion relation: general facts . . . . . . . . 16

5.3 Dispersion relation decomposed . . . . . . . 16

5.4 Scalar waves as a special case . . . . . . . . 17

5.5 A separable case . . . . . . . . . . . . . 17

5.6 Gauge conditions . . . . . . . . . . . 17

6 Gravitational waves in a metric model . . . . . . 18

6.1 Characteristic system . . . . . . . . . . 18

6.2 Waves in $\mathrm{GR}_{\|}$, the teleparallel equivalent of GR 19

7 Conclusions and outlook . . . . . . . . . . . . 19

Appendix: Young decomposition . . . . . . . . . . . 20

References . . . . . . . . . . . . . . . . 22

\section{Introduction}

Lately we followed in [18] the program of Kottler of 1922 to remove the metric tensor of spacetime, the gravitational potential within general relativity theory (GR), from the fun- 
damental laws of classical electromagnetism and gravity as far as possible. In particular, we applied this to the theory of gravity [31] in that we started from a translational gauge theory of gravity, also known as teleparallel theory of gravity (TG). ${ }^{1}$ We assume that our readers are familiar with [31]. The TG approach is reviewed in Blagojević et al. [8], see also Maluf [36], and Aldrovandi and Pereira [1]. For a somewhat related tetrad approach to gravity, in which affine symmetry is exploited, one should compare Sławianowski et al. [54].

The spacetime geometry of $\mathrm{TG}$ is represented by a four-dimensional (4d) manifold equipped with a coframe 1-form $\vartheta^{\alpha}=e_{i}^{\alpha} d x^{i}$ and with a linear connection 1form $\Gamma_{\alpha}^{\beta}=\Gamma_{i \alpha}{ }^{\beta} d x^{i}$, the curvature 2-form of which, $R_{\alpha}^{\beta}=\frac{1}{2} R_{i j \alpha}{ }^{\beta} d x^{i} \wedge d x^{j}$, vanishes globally: $R_{\alpha}{ }^{\beta} \equiv 0$. In the notation we follow basically the book [22]: $\alpha$ and $\beta$ are co- and contravariant frame indices, with $\alpha, \beta, \ldots=$ $\hat{0}, \hat{1}, \hat{2}, \hat{3}$, whereas $i, j, \ldots=0,1,2,3$ are coordinate indices.

Because of the vanishing curvature, we can pick suitable frames such that $\Gamma_{\alpha}{ }^{\beta}$ vanishes globally:

$\Gamma_{\alpha}^{\beta} \stackrel{*}{=} 0 \quad$ (everywhere in spacetime).

In this 'teleparallel gauge,' the covariant exterior derivative taken with respect to the connection $\Gamma_{\alpha}{ }^{\beta}$ is reduced to the ordinary exterior derivative. This will simplify our formalism. However, we will drop the star * over the equality sign in future since (1) is assumed to be valid throughout our paper.

The essence of the premetric approach can be formulated as follows. This universal field-theoretic scheme is based on conservation laws which hold true for the two types of variables: extensive fields ("how much?") and intensive ("how strong?") ones. These variables satisfy the fundamental equaions which are metric-free, whereas the metric comes in only via the linking equations which establish consitutive relations between the extensive and intensive variables.

Premetric electrodynamics [22] is based on the conservation laws of electric charge and magnetic flux which give rise to the fundamental equations $d H=J$ and $d F=0$. Here $H$ is the electromagnetic excitation 2-form (extensive variable) and $F$ the electromagnetic field strength 2-form (intensive variable). By introducing the constitutive relation $H=\kappa[F]$, one obtains a predictive physical theory.

The premetric gravity framework [31] can be constructed along the same lines by replacing the electric charge with a "gravitational charge" $\rightarrow$ mass $\rightarrow$ energy-momentum. In this introductory section, we provide a short overview of the premetric teleparallel approach.

${ }^{1}$ For the application of teleparallelism in continuum mechanics, see Delphenich [12].

\subsection{Field equations}

The field equations of TG will be our starting point. The inhomogeneous gravitational field equation of TG reads [31, Table I]

$d H_{\alpha}={ }^{(\vartheta)} \Sigma_{\alpha}+{ }^{(\mathrm{m})} \Sigma_{\alpha} \quad(4 \times 6$ components $)$.

It relates the twisted gravitational excitation 2-form

$H_{\alpha}=\frac{1}{2} H_{i j \alpha} d x^{i} \wedge d x^{j}=\frac{1}{2} H_{\beta \gamma \alpha} \vartheta^{\beta \gamma}=\frac{1}{2} \check{H}_{\alpha}^{\beta \gamma} \epsilon_{\beta \gamma}$

to its source, the sum of the twisted energy-momentum 3forms of gravity ${ }^{(\vartheta)} \Sigma_{\alpha}$ and of matter ${ }^{(\mathrm{m})} \Sigma_{\alpha}$, respectively. Here $\vartheta^{\beta \gamma}:=\vartheta^{\beta} \wedge \vartheta^{\gamma}$ and $\epsilon_{\beta \gamma}:=\frac{1}{2} \epsilon_{\beta \gamma \delta \varepsilon} \vartheta^{\delta} \wedge \vartheta^{\varepsilon}$, see [22, p. 39].

As was already pointed out in [17, p. 52], "...for consistency, we cannot allow spinning matter (other than as test particles) in such a $\mathrm{T}_{4} . .$. " that is, in a $4 \mathrm{~d}$ Weitzenböck spacetime. In other words, the field equation (2) is valid only for hydrodynamic and for electromagnetic matter. A careful proof of this stipulation was given by Obukhov and Pereira ${ }^{2}$ [47]. For matter with spin, the Dirac field, for example, the Lorentz group should also be gauged, which removes the teleparallelism constraint $R_{\alpha}{ }^{\beta}=0$. Then one arrives at a Poincaré gauge theory operating in a Riemann-Cartan spacetime with torsion and with Cartan curvature $R_{\alpha}{ }^{\beta} \neq 0$.

The homogeneous field equation of TG reads

$d F^{\alpha}=0 \quad(4 \times 6$ components $)$,

with the untwisted vector-valued 2-form $F^{\alpha}:=d \vartheta^{\alpha}$, the torsion of spacetime, which has the expansion

$F^{\alpha}=\frac{1}{2} F_{i j}^{\alpha} d x^{i} \wedge d x^{j}=\frac{1}{2} F_{\beta \gamma}{ }^{\alpha} \vartheta^{\beta \gamma}$.

Note that (4) is the first Bianchi identity of a linearly connected spacetime with vanishing curvature, see [53, Eq. (5.41)] or [22, Eq. (C.1.67)].

The analogy to the Maxwell equations of electrodynamics should be apparent, see [22]. Equation (2) represents four inhomogeneous Maxwell type equations and Eq. (4) four homogeneous Maxwell type equations. Since TG is a translational gauge theory, it has the four one-form potentials $\vartheta^{\alpha}=e_{i}^{\alpha} d x^{i}$, where four is the number of generators of the translation group.

\footnotetext{
${ }^{2}$ Within the framework of TG as a translational gauge theory, the coupling to matter is achieved via the minimal coupling procedure, strictly in the sense of a bona fide gauge theory. Dispensing with the minimal coupling principle (as Maluf [35] does, e.g.) is against the spirit of gauge theory.
} 


\subsection{Local and linear constitutive law}

In order to complete the two field equations of TG to a predictive system of equations, one has to adopt a constitutive law between excitation $H$ and field strength $F$. The simplest assumption is that the functional $H=\kappa[F]$ is local and linear,

$H_{\alpha}=\kappa_{\alpha \beta}\left[F^{\beta}\right]$.

To deduce the corresponding component representation, we remember that the functional $\kappa_{\alpha \beta}$ acts on 2-forms and creates as response other 2-forms. Since any 2-form can be decomposed with respect to the 2 -form basis $\vartheta^{v \rho}$, it is sufficient to study the behavior of $\vartheta^{\nu \rho}$ under the application of $\kappa_{\alpha \beta}$. Because of the assumed locality and linearity, we have

$\kappa_{\alpha \beta}\left[\vartheta^{\nu \rho}\right]=\frac{1}{2} \kappa_{\lambda \mu \alpha}{ }^{\nu \rho} \beta \vartheta^{\lambda \mu}$.

Let us come back to (6). We decompose $H_{\alpha}$ and $F^{\beta}$ and, by using (7), we find:

$$
\begin{aligned}
H_{\alpha} & =\frac{1}{2} H_{\lambda \mu \alpha} \vartheta^{\lambda \mu}=\kappa_{\alpha \beta}\left[F^{\beta}\right]=\kappa_{\alpha \beta}\left[\frac{1}{2} F_{\nu \rho}^{\beta} \vartheta^{\nu \rho}\right] \\
& =\frac{1}{2} F_{\nu \rho}^{\beta} \boldsymbol{\kappa}_{\alpha \beta}\left[\vartheta^{\nu \rho}\right]=\frac{1}{4} \kappa_{\lambda \mu \alpha}{ }^{\nu \rho} \beta F_{\nu \rho} \vartheta^{\lambda \mu} .
\end{aligned}
$$

By renaming some indices, we eventually derive the final formula

$H_{\beta \gamma \alpha}=\frac{1}{2} \kappa_{\beta \gamma \alpha}{ }^{\nu \rho}{ }_{\mu} F_{\nu \rho}{ }^{\mu}$.

see [31, Eq. (47)].

If we use Schouten's [53] notation $(\alpha \beta):=\frac{1}{2}\{\alpha \beta+\beta \alpha\}$ and, moreover, $[\alpha \beta]:=\frac{1}{2}\{\alpha \beta-\beta \alpha\}$, we have here the antisymmetries $H_{(\beta \gamma) \alpha}=0$ and $F_{(v \rho)}{ }^{\mu}=0$. Thus, the constitutive tensor obeys the identities

$\kappa_{(\beta \gamma) \alpha}{ }^{\nu \rho}{ }_{\mu}=0$ and $\kappa_{\beta \gamma \alpha}{ }^{(\nu \rho)}{ }_{\mu}=0$.

Accordingly, $\kappa_{\beta \gamma \alpha}{ }^{\nu \rho}{ }_{\mu}$ has $(6 \times 4)^{2}=576$ independent components. In the corresponding electrodynamics case, the constitutive law reads $H_{\beta \gamma}=\frac{1}{2} \kappa_{\beta \gamma}{ }^{\nu \rho} F_{\nu \rho}$. Thus, by contrast, we have only $6^{2}=36$ independent components. As we will see further down, if we study only reversible processes, then this number is appreciably downsized in both cases.

A concise Hamiltonian formulation of teleparallel gravity was given by Ferraro and Guzmán [14]. Recently Hohmann et al. [26] studied teleparallel gravity, but instead of taking local and linear constitutive equations, they turned to local and nonlinear ones in order to incorporate $f(T)$ theories into the general TG formalism. These investigations [26] are very helpful since they bring order into the plethora of $f(T)$-theories and make them more transparent. Such nonlinear models provide an interesting development of gravitational theory based on an analogy with Born-InfeldPlebański electrodynamics. We believe, though, that there is, at the present time, no real need to push nonlinear constitutive laws, since gravity is nonlinear anyway, due to its self-interaction - and this in spite of a linear constitutive law, which guarantees the quasi-linearity of the emerging field equation.

Kostelecký and Mewes [33] investigated Lorentz and diffeomorphism violations in linearized gravity. In this context, they introduced tensors which are of a similar type as our constitutive tensor $\chi$. Our group-theoretical treatment of $\chi$ in Sect. 3 is reminiscent of their method. However, in our article the full nonlinearity of gravity is treated in a premetric framework.

We would like to stress the following fact about teleparallelism theories: We have always a frame $e_{\alpha}=e^{i}{ }_{\alpha} \partial_{i}$ and a coframe $\vartheta^{\beta}=e_{j}^{\beta} d x^{j}$ with us, that is, we can always change from holonomic to anholonomic indices by using $e^{i}{ }_{\alpha}$ and $e_{j}{ }^{\beta}$, respectively - and vice versa. This implies that all the indices in premetric TG are fundamentally equal, in particular, all those occurring in $\kappa_{\beta \gamma \alpha}{ }^{\nu \rho}{ }_{\mu}$ in (9). The 'group' indices $\alpha$ and $\mu$ are of the same quality as the 'form' indices $\beta, \gamma, \nu, \rho$. Contractions with all indices are always allowed. Of course, at the premetric stage, raising and lowering of indices is only possible with the totally antisymmetric LeviCivita symbols $\epsilon_{\alpha \beta \gamma \delta}= \pm 1,0$ and $\epsilon^{\mu \nu \rho \sigma}=\mp 1,0$, since no metric is available so far in our premetric framework.

If we follow the pattern of electrodynamics as formulated in tensor calculus, see Post [50], then it is obvious that we should introduce the excitation with the components $\check{H}^{\beta \gamma}{ }_{\alpha}=\frac{1}{2} \epsilon^{\beta \gamma \mu \nu} H_{\mu \nu \alpha}$, as already defined in Eq. (3). Thus,

$\check{H}^{\beta \gamma}{ }_{\alpha}=\frac{1}{2} \chi^{\beta \gamma}{ }_{\alpha}{ }^{\nu \rho}{ }_{\mu} F_{\nu \rho}{ }^{\mu}$,

with

$\chi^{\beta \gamma_{\alpha}{ }^{\nu \rho}{ }_{\mu}}:=\frac{1}{2} \epsilon^{\beta \gamma \delta \varepsilon} \kappa_{\delta \varepsilon \alpha}{ }^{\nu \rho}{ }_{\mu}$.

The constitutive tensor density ${ }^{3} \chi^{\beta \gamma}{ }_{\alpha}{ }^{\nu \rho}{ }_{\mu}$ is equivalent to $\kappa_{\beta \gamma \alpha}{ }^{\nu \rho}{ }_{\mu}$, in particular,

$\chi^{(\beta \gamma)}{ }_{\alpha}^{\nu \rho}{ }_{\mu}=0$ and $\chi^{\beta \gamma_{\alpha}{ }^{(\rho v)}{ }_{\mu}=0 .}$

But there is still a third useful version of the constitutive tensor available. For the purpose of the irreducible decomposition under the $\operatorname{GL}(4, \mathbb{R})$, it is optimal to have the indices of the constitutive tensor all exclusively either in

\footnotetext{
${ }^{3}$ This tensor density emerged already in [21], see also [1,9].
} 
lower or in upper position. Since so far we have no metric at our disposal, we can only move the antisymmetric sets of indices. If we have a look at (9), we can rewrite it with $\check{F}^{v \rho \mu}=\frac{1}{2} \epsilon^{\nu \rho v^{\prime} \rho^{\prime}} F_{\nu^{\prime} \rho^{\prime}} \mu$ as

$H_{\beta \gamma \alpha}=\frac{1}{2} \check{\chi}_{\beta \gamma \alpha \nu \rho \mu} \check{\boldsymbol{F}}^{\nu \rho \mu}$,

that is,

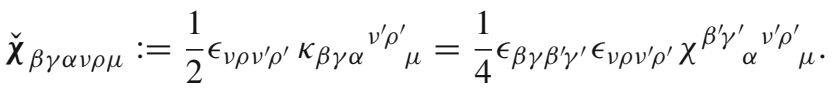

We have the symmetries

$\check{\chi}_{(\beta \gamma) \alpha v \rho \mu}=0$ and $\check{\chi}_{\beta \gamma \alpha(v \rho) \mu}=0$.

\subsection{Reversibility}

A process which can in no way be completely reversed is termed irreversible, all other processes reversible. That a process may be irreversible, it is not sufficient that it cannot be directly reversed. This is the case with many mechanical processes which are not irreversible... ...The full requirement is, that it be impossible, even with the assistance of all agents in nature, to restore everywhere the exact initial state when the process has once taken place... the generation of heat by friction, the expansion of a gas without the performance of external work and the absorption of external heat, the conduction of heat, etc., are irreversible processes...

Max Planck [49, p. 84] ${ }^{4}$

The merit of formulating a field theory only in terms of its field equations - here Eqs. (2) and (4) - and an associated constitutive law - here Eq. (6) - is that it covers both processes, irreversible and reversible ones. At first, like in the conventional treatment of general relativity [13], we turn our attention to reversible processes. From their definition it is clear that, for instance, periodic processes and those the equations of motion of which are formulated in a time symmetric way, are reversible. Time symmetry means that we can substitute in the field equations $t$ by $-t$ without changing them.

Thus dissipation is not allowed in reversible processes and we can define for each such process an energy function and,

\footnotetext{
${ }^{4}$ Similarly, we have: Suppose that when a system under consideration changes from a state, $\alpha$, to another state, $\alpha^{\prime}$, the environment changes from $\beta$ to $\beta^{\prime}$. If in some way it is possible to return the system from $\alpha^{\prime}$ to $\alpha$ and at the same time to return the environment from $\beta^{\prime}$ to $\beta$, the process $(\alpha, \beta) \rightarrow\left(\alpha^{\prime}, \beta^{\prime}\right)$ is said to be reversible. Ryogo Kubo [34, $\mathrm{p}$. $61]$.
}

by a Legendre transformation, a Lagrangian. Accordingly, reversible processes can always be formulated by means of an action principle. For TG, the twisted Lagrange 4-form reads

${ }^{(\vartheta)} \Lambda=-\frac{1}{2} F^{\alpha} \wedge H_{\alpha}=-\frac{1}{2} F^{\alpha} \wedge \kappa_{\alpha \beta}\left[F^{\beta}\right]$.

We substitute (5) and (8) into (17) and find:

$$
\begin{aligned}
{ }^{(\vartheta)} \Lambda & =-\frac{1}{2}\left(\frac{1}{2} F_{\eta \zeta^{\alpha}} \vartheta^{\eta \zeta}\right) \wedge\left(\frac{1}{4} \kappa_{\lambda \mu \alpha}{ }^{\nu \rho}{ }_{\beta} F_{\nu \rho}{ }^{\beta} \vartheta^{\lambda \mu}\right) \\
& =-\frac{1}{16} \kappa_{\lambda \mu \alpha}{ }^{\nu \rho}{ }_{\beta} F_{\eta \zeta}{ }^{\alpha} F_{\nu \rho}{ }^{\beta} \vartheta^{\eta \zeta \lambda \mu} .
\end{aligned}
$$

With the definition (12) and the volume 4-form vol, Eq. (18) can be rewritten as

${ }^{(\vartheta)} \Lambda=-\frac{1}{8} \chi^{\beta \gamma}{ }_{\alpha}{ }^{v \rho}{ }_{\mu} F_{\beta \gamma}{ }^{\alpha} F_{\nu \rho}{ }^{\mu}$ vol

or, by renaming the indices of the $F$ 's, equivalently as

${ }^{(\vartheta)} \Lambda=-\frac{1}{8} \chi^{\nu \rho}{ }_{\mu}^{\beta \gamma}{ }_{\alpha} F_{\beta \gamma}{ }^{\alpha} F_{v \rho}{ }^{\mu}$ vol.

Consequently, only those components of the constitutive tensor enter the Lagrangian that satisfy the relations

$\chi^{\beta \gamma}{ }_{\alpha}{ }^{\nu \rho}{ }_{\mu}=\chi^{\nu \rho}{ }_{\mu}^{\beta \gamma}{ }_{\alpha}$.

If we assume that the model is completely specified by the Lagrangian (17), we restrict our considerations to reversible processes. Then the relations (21) are necessary conditions, which correspond to the symmetry of a $24 \times 24$ matrix. Thus, the set of the 576 independent components of $\chi^{\beta \gamma_{\alpha}}{ }^{\nu \rho}{ }_{\mu}$ reduces to only 300 ones.

It is possible to rewrite the constitutive law in a more compact way. We introduce the 6-dimensional co-basis $\vartheta^{\alpha \beta}$ $\rightarrow \vartheta^{I}$ in the space of 2-forms, with the collective indices $I, J, \cdots=(01,02,03 ; 23,31,12)=(1,2, \ldots, 6)$, see $[22$, p. 40]. Then excitation and field strength decompose as $H_{\alpha}=$ $H_{I \alpha} \vartheta^{I}$ and $F^{\beta}=F_{J}^{\beta} \vartheta^{J}$, respectively, and the constitutive law reads,

$H_{I \alpha}=\kappa_{I \alpha}{ }^{J}{ }_{\beta} F_{J}{ }^{\beta}, \quad \check{H}_{\alpha}^{I}=\frac{1}{2} \chi_{\alpha}^{I}{ }_{\alpha}^{J} F_{J}{ }^{\beta}$,

with the 300 independent components

$\chi_{\alpha}^{I}{ }_{\alpha}^{J}=\chi^{J}{ }_{\beta}^{I}$.

Thus, for the Lagrangian we find

${ }^{(\vartheta)} \Lambda=-\frac{1}{2} \chi^{I}{ }_{\alpha}^{J}{ }_{\beta} F_{I}^{\alpha} F_{J}^{\beta}$ vol. 
This closes our short introduction to TG. Like all classical theories, whose form is established, we can put TG into a Tonti type diagram [56] in order to clearly display its structure. This will be done for the first time in Sect. 2. Then we turn to a closer examination of the constitutive tensor of TG. In Sect. 3, we decompose it into smaller pieces, in particular into the irreducible pieces with respect to the linear group $\operatorname{GL}(4, \mathbb{R})$. In Sect. 4, metric dependent constitutive tensors will be addressed, in particular those which relate TG to general relativity. In Sect. 5, we will study the propagation of gravitational waves in TG within the geometric optics approximation. We will follow the procedure that we developed for electrodynamics [2,22,29]. Since in TG we have four generators of the gauge group, things become a bit more complicated than in electrodynamics. In Sect. 6, we will specialize these considerations on gravitational waves to metric dependent models.

\section{Tonti diagram of the premetric teleparallel theory of gravity}

Over the past decades, Tonti [56] developed a general classification program for classical and relativistic theories in physics, such as, e.g., for particle dynamics, electromagnetism, the mechanics of deformable media, fluid mechanics, thermodynamics, and gravitation. Here we will display in Fig. 1 for the first time an appropriate and consistent diagram of the teleparallel theory of gravity (TG).

If a theory is well-understood, its configuration and its source variables can be clearly identified and their interrelationships displayed in the form of a Tonti diagram. Such a diagram defines what one may call the skeleton of a theory. In Tonti's book [56], for all classical theories, including the relativistic ones, a corresponding framework was established - and this step by step, based on an operational definition of the quantities involved.

Tonti [56, p. 402] has also displayed a diagram for relativistic gravitation. In Tonti's own words, it was an "attempt" of a diagram based on an ansatz for a tetrad theory of gravity by Kreisel and Treder, see [57, pp. 60-67, pp. 71-91]. Due to our enhanced knowledge of TG, see [8, Chapters 5 and 6], we can now improve on Tonti's attempt, see [23] and our Fig. 1. The notation in Fig. 1 is based on our recent paper [31] and on the present one.

Let us have a look at our new diagram. The configuration variables of TG are the coordinates $x^{i}$ of the 4-dimensional spacetime (four 0 -forms) and the coframes $\vartheta^{\alpha}$ (four 1 -forms). By differentiation, we find the torsion $F^{\beta}$ (four 2-forms) and by further differentiation the homogeneous field equation of gravity $d F^{\beta}=0$ (four 3-forms), the right hand side of which vanishes.
The round boxes on the left column depict geometrical objects and the square boxes interrelate these geometrical objects. The formula $d F^{\beta}=0$, for example, corresponds to the first Bianchi identity of a teleparallel spacetime. In a Riemann-Cartan space, we have $D F^{\beta}=\vartheta^{\alpha} \wedge R_{\alpha}^{\beta}$. In teleparallelism, the curvature vanishes, $R_{\alpha}{ }^{\beta}=0$, and, in the teleparallel gauge, $d F^{\beta}=0$.

For global variables, Tonti distinguishes spatial domains, such as volumes $\mathbf{V}$, surfaces $\mathbf{S}$, lines $\mathbf{L}$, and points $\mathbf{P}$, with respect to time he introduces instants $\mathbf{I}$ and intervals $\mathbf{T}$. Furthermore, for the respective domains, he has inner (interior) - and outer (exterior) $\sim$ orientation.

Hence $[\widetilde{\mathbf{I}} \times \overline{\mathbf{S}}]$, for example, refers to a time domain $\widetilde{\mathbf{I}}$ with exterior orientation and a space domain $\overline{\mathbf{S}}$ with interior orientation. And this domain $[\tilde{\mathbf{I}} \times \overline{\mathbf{S}}]$ supports the torsion two-form. The holonomic coordinates $x^{j}$, to take another example, depend on an instant of time I and a spatial point P. i.e. $[\mathbf{I} \times \mathbf{P}]$. Then we have to add the orientation. All exterior forms on the left columns are forms without twist, see [22], those on the right columns all carry twist. This can be read off from the corresponding orientations. This comes about as follows.

For configuration variables the associated space elements are endowed always with an interior orientation, whereas in the case of source variables it is the exterior orientation which plays a role. This behavior is found by phenomenologically examining the different theories. According to Tonti, the underlying theoretical reason for this correspondence is not clear, but phenomenology does not allow any other attributions. The configuration variables are related to the theory of chains of algebraic topology, whereas the source variables are associated to co-chains. For more details, we refer to the exhaustive monograph of Tonti [56].

Since the gravitational field itself carries energy-momentum, it is also the source of a new gravitational field, which likewise carries energy-momentum, etc. Thus, like general relativity, TG is an intrinsically nonlinear theory, even it carries a linear constitutive law. Within the field equation of TG, $d H_{\alpha}-{ }^{(\vartheta)} \Sigma_{\alpha}={ }^{(\mathrm{m})} \Sigma_{\alpha}$, the gravitational energy-momentum 3-form

$\left.\left.{ }^{(\vartheta)} \Sigma_{\alpha}:=\frac{1}{2}\left[F^{\beta} \wedge\left(e_{\alpha}\right\rfloor H_{\beta}\right)-H_{\beta} \wedge\left(e_{\alpha}\right\rfloor F^{\beta}\right)\right]$

shows up explicitly and is a manifestation of this nonlinearity. By differentiation of the field equation, we find

$d d H_{\alpha}=0=d\left({ }^{(\vartheta)} \Sigma_{\alpha}+{ }^{(\mathrm{m})} \Sigma_{\alpha}\right)$.

Thus, $d^{(\mathrm{m})} \Sigma_{\alpha}=-d^{(\vartheta)} \Sigma_{\alpha}$ is nonvanishing in general, which clearly shows up in our Tonti diagram.

The Tonti diagram displayed in Fig. 1 was constructed for the premetric version of teleparallelism, no metric is involved 
at all. We developed the corresponding formalism in our previous paper in [31, Sec.II]. Above, in Sect. 1.1, we discussed already that teleparallel gravity is only equivalent to GR as long as the matter which is involved does not carry intrinsic spin. If this is the case, the energy-momentum of matter is described by the 3 -form ${ }^{(\mathrm{m})} \sigma_{\alpha}$, with $\vartheta_{[\alpha} \wedge^{(\mathrm{m})} \sigma_{\beta]}=0$, i.e., the corresponding energy-momentum tensor is symmetric. Since $\vartheta_{\alpha}:=g_{\alpha \gamma} \vartheta^{\gamma}$, we need a metric for the specification of such an energy-momentum ${ }^{(\mathrm{m})} \sigma_{\alpha}$. And the metric $g_{\alpha \beta}$ induces an associated Levi-Civita connection 1-form $\widetilde{\Gamma}_{\alpha}{ }^{\beta}$.

The energy-momentum law, in the teleparallel gauge, reads $\left.d^{(\mathrm{m})} \Sigma_{\alpha}=\left(e_{\alpha}\right\rfloor F^{\beta}\right) \wedge{ }^{(\mathrm{m})} \Sigma_{\beta}$. If we dispense with the teleparallel gauge for the moment, the derivative $d^{(\mathrm{m})} \Sigma_{\alpha}$ can be substituted by $D^{(\mathrm{m})} \Sigma_{\alpha}$, with $D$ as the covariant exterior derivative operator with respect to the teleparallel connection $\Gamma_{\alpha}{ }^{\beta}$. Having now a metric available, we can assume that the teleparallel connection is metric compatible, that is, $D g_{\alpha \beta}=0$. For the symmetric energy-momentum, we have then the energy-momentum law

$\left.D^{(\mathrm{m})} \sigma_{\alpha}=\left(e_{\alpha}\right\rfloor F^{\beta}\right) \wedge^{(\mathrm{m})} \sigma_{\beta}$.

Due to a lemma of Meyer [38], the right side of (27) can be absorbed by the left side. This can be demonstrated by expanding the covariant derivative:

$\left.d^{(\mathrm{m})} \sigma_{\alpha}-\left(\Gamma_{\alpha}{ }^{\beta}+e_{\alpha}\right\rfloor F^{\beta}\right) \wedge^{(\mathrm{m})} \sigma_{\beta}=0$.

Let us now introduce a tilde to denote the Riemannian objects and operators: $\widetilde{\Gamma}_{\alpha}{ }^{\beta}$ is the Christoffel connection, for example, and $\widetilde{D}$ the Riemannian covariant derivative. Since $\Gamma_{\alpha}{ }^{\beta}=$ $\widetilde{\Gamma}_{\alpha}{ }^{\beta}-K_{\alpha}{ }^{\beta}$, with the contortion 1 -form $K_{\alpha}{ }^{\beta}$, we can rewrite (28) as

$\left.\widetilde{D}^{(\mathrm{m})} \sigma_{\alpha}-\left(-K_{\alpha}^{\beta}+e_{\alpha}\right\rfloor F^{\beta}\right) \wedge^{(\mathrm{m})} \sigma_{\beta}=0$.

The contortion, is related to the torsion via $F^{\beta}=K^{\beta}{ }_{\gamma} \wedge \vartheta^{\gamma}$. If we substitute this into (29), it can be recast into

$\left.\widetilde{D}^{(\mathrm{m})} \sigma_{\alpha}+\left[K_{\alpha}{ }^{\beta}-\left(e_{\alpha}\right\rfloor K^{\beta}{ }_{\gamma}\right) \vartheta^{\gamma}+K_{\gamma}^{\beta}{ }_{\gamma} \delta_{\alpha}^{\gamma}\right] \wedge^{(\mathrm{m})} \sigma_{\beta}=0$.

Because of the antisymmetry of the contortion, $K_{(\alpha \beta)}=0$, Eq. (30) simplifies to

$\left.\widetilde{D}^{(\mathrm{m})} \sigma_{\alpha}-\left(e_{\alpha}\right\rfloor K^{\beta \gamma}\right) \vartheta_{[\gamma} \wedge^{(\mathrm{m})} \sigma_{\beta]}=0$.

Now we recall that ${ }^{(\mathrm{m})} \sigma_{\alpha}$ is symmetric, $\vartheta_{[\gamma} \wedge{ }^{(\mathrm{m})} \sigma_{\beta]}=0$. Consequently,

$\widetilde{D}^{(\mathrm{m})} \sigma_{\alpha}=0$
Tonti diagram of the premetric teleparallel theory of gravity ${ }^{\star}$ (TG)

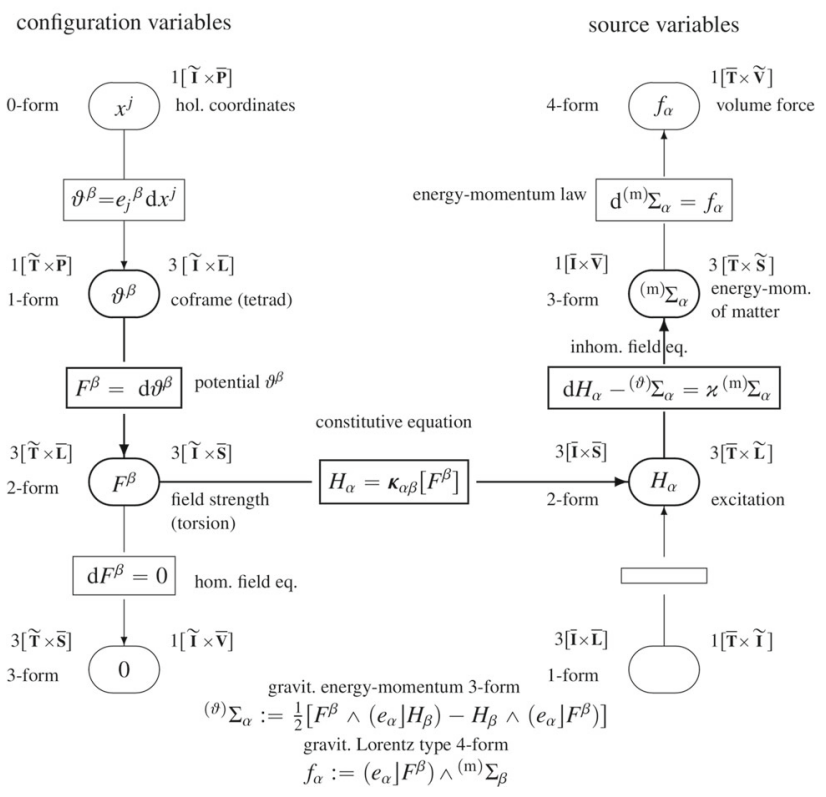

Fig. 1 Patterned after E. Tonti: The Mathematical Structure of Classical and Relativistic Physics, A general classification diagram (Birkhäuser-Springer, New York, 2013) pages 402 and 315. Notation: Y. Itin, F. W. Hehl, Yu. N. Obukhov, Phys. Rev. D 95, 084020 (2017), arXiv:1611.05759. We denoted here the torsion 2-form with $F^{\beta}$ in order to underline its function as a field strength. Usually, however, we use for torsion $T^{\beta}$.-we chose everywhere the 'teleparallel gauge' such that the connection 1-form vanishes globally: $\Gamma^{\alpha \beta}(x) \stackrel{*}{=} 0$. For the reversible case, we have the gravitational Lagrangian as ${ }^{(\vartheta)} \Lambda=-\frac{1}{2} F^{\alpha} \wedge H_{\alpha}$ and $\Lambda={ }^{(\vartheta)} \Lambda+{ }^{(\mathrm{m})} \Lambda$. The gravitational constant is denoted by $\varkappa$. ${ }^{\star}$ Also known as translation gauge theory of gravity

This is the energy-momentum law of GR. Accordingly, our entry $\left.d^{(\mathrm{m})} \Sigma_{\alpha}=\left(e_{\alpha}\right\rfloor F^{\beta}\right) \wedge{ }^{(\mathrm{m})} \Sigma_{\beta}$ in the Tonti diagram becomes, provided a symmetric energy-momentum tensor is prescribed, $\widetilde{D}^{(\mathrm{m})} \sigma_{\alpha}=0$, well in accordance with the vanishing divergences in analogous Tonti diagrams.

\section{Decompositions of the constitutive tensor}

In this section we characterize the constitutive tensor due to its symmetry transformations relative to the general linear group $\mathrm{GL}(4, \mathbb{R})$ and the permutation (symmetry) group. We refer to Hamermesh [15] and to Barut and Raczka [5] as background information for group theory.

\subsection{Two forms of the constitutive tensor}

According to (11), the constitutive tensor density $\chi^{\alpha \beta}{ }_{\mu}{ }^{\gamma \delta}{ }_{\nu}$ is a order- $\left(\begin{array}{l}4 \\ 2\end{array}\right)$ tensor density that is skew-symmetric in the two pairs of upper indices, see (13): 
$\chi_{\mu}^{(\alpha \beta)}{ }_{v}^{\gamma \delta}=0, \quad \chi_{\mu}^{\alpha \beta}{ }_{v}^{(\gamma \delta)}=0$.

In $n$ dimensions, we have $n\left(\begin{array}{l}n \\ 2\end{array}\right) n\left(\begin{array}{l}n \\ 2\end{array}\right)=\frac{1}{4} n^{4}(n-1)^{2}$ independent components. For $n=4$, it gives 576 relevant components. Due to this large number, it is instructive to consider decomposition of the constitutive tensor into smaller pieces. We apply Young's decomposition technique that yields irreducible decomposition under the group $\operatorname{GL}(4, \mathbb{R})$. For our conventions and the details of the Young decomposition, see "Appendix". For the tensor $\chi^{\alpha \beta} \mu^{\gamma \delta}{ }_{\nu}$, separate $S_{4}$-permutations of four upper indices and $S_{2}$ permutations of two lower indices are available, where $S_{n}$ denotes the $n$-dimensional permutation group. Correspondingly, we are dealing with the Cartesian product group $S_{4} \times S_{2}$.

Although the metric tensor is not available in our construction, we can, as we have shown in (15), lower two pairs of skew-symmetric indices by the Levi-Civita symbol. Thus, we have a order- $\left(\begin{array}{l}0 \\ 6\end{array}\right)$ tensor

$\check{\chi}_{\alpha \beta \mu \gamma \delta v}:=\frac{1}{4} \epsilon_{\alpha \beta \omega \lambda} \epsilon_{\gamma \delta \rho \sigma} \chi_{\mu \lambda}^{\omega \sigma}{ }_{\nu}$,

with the identities

$\check{\chi}_{(\alpha \beta) \mu \gamma \delta v}=0, \quad \check{\chi}_{\alpha \beta \mu(\gamma \delta) v}=0$.

This tensor is naturally decomposed under the permutation group $S_{6}$. Both types of decompositions are invariant under the action of the basis transformation group $\operatorname{GL}(4, \mathbb{R})$.

\section{$3.2 S_{4} \times S_{2}$ decomposition of $\chi^{\alpha \beta}{ }_{\mu}^{\gamma \delta}{ }_{\nu}$}

Treating the covariant and contravariant indices as belonging to two separate tensor spaces, the irreducible decomposition of $\chi^{\alpha \beta}{ }_{\mu}^{\gamma \delta}$ is defined as a product of the irreducible decompositions. The corresponding Young diagrams are expanded as

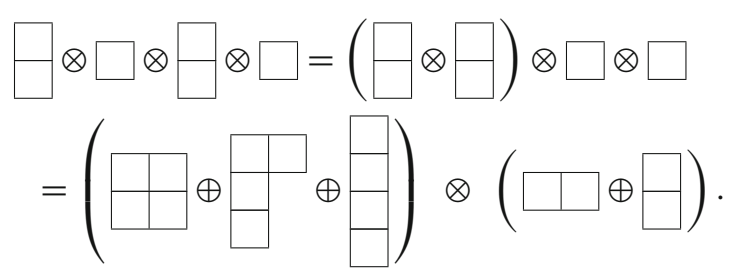

Here, the three first diagrams relate to the upper indices. This decomposition repeats the known irreducible decomposition of the electromagnetic constitutive tensor. The remaining two diagrams represent the symmetric and antisymmetric parts of the second order tensor. Collecting all possible combinations, we obtain the decomposition of the tensor $\chi^{\alpha \beta} \mu^{\gamma \delta}{ }_{v}$ into 6 independent pieces:
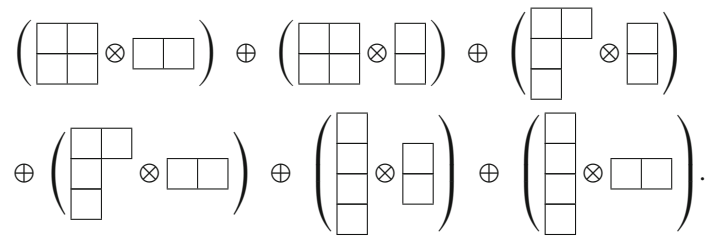

In tensor notation, the above decomposition reads

$\chi_{\mu}^{\alpha \beta}{ }_{\mu}^{\gamma \delta}{ }_{\nu}=\sum_{I=1}^{6}{ }^{[I]} \mathbb{P}_{\omega \lambda \mu \rho \sigma \nu}^{\alpha \beta \kappa \gamma \delta \epsilon} \chi^{\omega \lambda}{ }_{\kappa}^{\rho \sigma}{ }_{\epsilon}=\sum_{I=1}^{6}{ }^{[I]} \chi^{\alpha \beta}{ }_{\mu}^{\gamma \delta}{ }_{\nu}$.

Here we used the set of six projection operators ${ }^{[I} \mathbb{P}$. We chose the labeling of $I=1, \ldots, 6$ such that it corresponds to the same sequence of Young diagrams as depicted in the second equality of Eq. (38). Explicitly, the $S_{4} \times S_{2}$-irreducible pieces of the constitutive tensor are expressed as

${ }^{[1]} \chi^{\alpha \beta}{ }_{\mu}{ }^{\gamma \delta}{ }_{v}:=\chi^{\alpha \beta}\left(\mu^{\gamma \delta}{ }_{\nu)}-{ }^{[3]} \chi^{\alpha \beta} \mu^{\gamma \delta}{ }_{v}-{ }^{[5]} \chi^{\alpha \beta}{ }_{\mu}{ }^{\gamma \delta}{ }_{\nu}\right.$,

${ }^{[2]} \chi^{\alpha \beta}{ }_{\mu}^{\gamma \delta}{ }_{v}:=\chi^{\alpha \beta}{ }_{[\mu}^{\gamma \delta}{ }_{\nu]}-{ }^{[4]} \chi^{\alpha \beta}{ }_{\mu}^{\gamma \delta}{ }_{v}-{ }^{[6]} \chi^{\alpha \beta}{ }_{\mu}{ }^{\gamma \delta}{ }_{\nu}$,

$\left.{ }^{[3]} \chi^{\alpha \beta}{ }_{\mu}^{\gamma \delta}{ }_{v}:=\frac{1}{2}\left(\chi^{\alpha \beta}\left(\mu^{\gamma \delta}{ }_{\nu}\right)-\chi^{\gamma \delta}{ }_{(\mu}{ }^{\alpha \beta}{ }_{\nu}\right)\right)$,

${ }^{[4]} \chi^{\alpha \beta}{ }_{\mu}^{\gamma \delta}{ }_{\nu}:=\frac{1}{2}\left(\chi^{\alpha \beta}{ }_{\left[\mu^{\gamma \delta}{ }_{\nu]}\right.}-\chi^{\gamma \delta}{ }_{[\mu}^{\alpha \beta}{ }_{\nu]}\right)$,

${ }^{[5]} \chi^{\alpha \beta}{ }_{\mu}^{\gamma \delta}{ }_{v}:=\chi^{[\alpha \beta}\left(\mu^{\gamma \delta]}{ }_{v)}\right.$,

${ }^{[6]} \chi^{\alpha \beta}{ }_{\mu}^{\gamma \delta}{ }_{v}:=\chi^{[\alpha \beta}\left[\mu^{\gamma \delta]} v\right]$.

In terms of independent components in $n=4$ dimensions, this decomposition corresponds to

$576=200 \oplus 120 \oplus 150 \oplus 90 \oplus 10 \oplus 6$.

It is straightforward to show that the above operators ${ }^{[I]} \mathbb{P}={ }^{[I]} \mathbb{P}_{\omega \lambda \mu \rho \sigma \nu}^{\alpha \beta \kappa \gamma \delta \epsilon}$ are orthogonal projectors:

${ }^{[I]} \mathbb{P} \circ{ }^{[J]} \mathbb{P}={ }^{[I]} \mathbb{P} \delta^{I J}, \quad \delta^{I J}=\left\{\begin{array}{ll}1 & \text { for } I=J \\ 0 & \text { for } I \neq J\end{array}\right.$.

In (39)-(44), we first decompose the gravitational constitutive tensor into three independent pieces relative to its four upper indices. This $S_{4}$ decomposition is done completely similar to electrodynamics. Then we extract relative to the pair of the lower indices the symmetric and antisymmetric parts. Since the principal and axion part of the electromagnetic constitutive tensor are reversible, the corresponding parts of the gravitational constitutive tensor are reversible when symmetrized in the lower indices. The electrodynamics the skewon part is irreversible. Thus its gravitational analog is reversible when antisymmetrized in the lower indices. The 
remaining three parts are irreversible (change their sign under permutation of triads of indices).

In order to demonstrate this behavior explicitly, we decompose the constitutive tensor into its reversible and irreversible parts,

$\chi^{\alpha \beta}{ }_{\mu}^{\gamma \delta}{ }_{v}=\stackrel{+}{\chi}^{\alpha \beta}{ }_{\mu}{ }^{\gamma \delta}{ }_{v}+\bar{\chi}^{\alpha \beta}{ }_{\mu}{ }^{\gamma \delta}{ }_{\nu}$,

with

$\stackrel{+}{\chi}^{\alpha \beta}{ }_{\mu}{ }^{\gamma \delta}{ }_{\nu}:=\frac{1}{2}\left(\chi^{\alpha \beta}{ }_{\mu}^{\gamma \delta}{ }_{\nu}+\chi^{\gamma \delta}{ }_{\nu}^{\alpha \beta}{ }_{\mu}\right)=\stackrel{+}{\chi}^{\gamma \delta}{ }_{\nu}{ }^{\alpha \beta}{ }_{\mu}$,

$\bar{\chi}^{\alpha \beta}{ }_{\mu}^{\gamma \delta}{ }_{\nu}:=\frac{1}{2}\left(\chi^{\alpha \beta}{ }_{\mu}^{\gamma \delta}{ }_{\nu}-\chi^{\gamma \delta}{ }_{\nu}^{\alpha \beta}{ }_{\mu}\right)=-\bar{\chi}^{\gamma \delta}{ }_{\nu}^{\alpha \beta}{ }_{\mu}$.

The first part ${ }^{+}{ }^{\alpha \beta}{ }_{\mu}{ }^{\gamma \delta}{ }_{\nu}$ describes reversible processes, it can be derived from a Lagrangian. In contrast, the second part $\bar{\chi}^{\alpha \beta}{ }_{\mu}{ }^{\gamma \delta}{ }_{\nu}$ corresponds to irreversibility, and it does not enter the Lagrangian. Following the nomenclature in electrodynamics, we will call $\bar{\chi}^{\alpha \beta}{ }_{\mu}^{\gamma \delta}{ }_{v}$ the skewon part.

In the next step, we can decompose each of these parts into the symmetric/antisymmetric piece in the lower indices:

$\stackrel{ \pm}{\chi}^{\alpha \beta}{ }_{\mu}{ }^{\gamma \delta}{ }_{\nu}=\stackrel{ \pm}{\chi}^{\alpha \beta}{ }_{(\mu}{ }^{\gamma \delta}{ }_{\nu)}+\stackrel{ \pm}{\chi}^{\alpha \beta}{ }_{[\mu}{ }^{\gamma \delta}{ }_{\nu]}$.

Two immediate consequences are

$\stackrel{+}{\chi}_{[\alpha \beta}^{[\mu \delta \delta]}{ }_{\nu]}^{\gamma \delta 0,} \bar{\chi}^{[\alpha \beta}{ }_{(\mu}^{\gamma \delta]}{ }_{v)} \equiv 0$.

Thus, axion pieces can be extracted only from $\stackrel{+}{\chi}^{\alpha \beta}{ }_{(\mu}{ }^{\gamma \delta}{ }_{\nu)}$ and from $\bar{\chi}^{\alpha \beta}{ }_{[\mu}^{\gamma \delta}{ }_{\nu]}$.

After these preliminaries, we can recast the irreducible decompositions (39)-(44) into a more transparent form,

${ }^{[1]} \chi^{\alpha \beta}{ }_{\mu}^{\gamma \delta}{ }_{\nu}:=\stackrel{+}{\chi}^{\alpha \beta}\left(\mu^{\gamma \delta}{ }_{\nu}-\stackrel{+}{\chi}^{[\alpha \beta}{ }_{(\mu}^{\gamma \delta]}{ }_{\nu)}\right.$,

${ }^{[2]} \chi^{\alpha \beta}{ }_{\mu}^{\gamma \delta}{ }_{v}:=\bar{\chi}^{\alpha \beta}{ }_{[\mu}^{\gamma \delta}{ }_{\nu]}-\bar{\chi}^{[\alpha \beta}{ }_{[\mu}^{\gamma \delta]}{ }_{\nu]}$,

${ }^{[3]} \chi^{\alpha \beta}{ }_{\mu}^{\gamma \delta}{ }_{v}:=\bar{\chi}^{\alpha \beta}{ }_{(\mu}^{\gamma \delta}{ }_{\nu)}$,

${ }^{[4]} \chi^{\alpha \beta}{ }_{\mu}^{\gamma \delta}{ }_{v}:=\stackrel{+}{\chi}^{\alpha \beta}{ }_{[\mu}{ }^{\gamma \delta}{ }_{\nu]}$,

${ }^{[5]} \chi_{\mu}^{\alpha \beta}{ }_{\mu}^{\gamma \delta}:=\stackrel{+}{\chi}^{[\alpha \beta}\left(\mu^{\gamma \delta]} v\right)$,

${ }^{[6]} \chi^{\alpha \beta}{ }_{\mu}^{\gamma \delta}{ }_{\nu}:=\bar{\chi}^{[\alpha \beta}{ }_{[\mu}^{\gamma \delta]}{ }_{\nu]}$.

In other words, we have a decomposition of the reversible and skewon parts into, respectively,
Table 1 Physical identifications of the irreducible parts of the constitutive tensor $\chi^{\alpha \beta}{ }^{\mu \nu}{ }_{\rho}$

\begin{tabular}{lllll}
\hline Irr. parts & Nomenclature & Lagr. & En-mom. & Disp. \\
\hline${ }^{[1]} \chi^{\alpha \beta} \gamma^{\mu v}{ }_{\rho}$ & Principal-1 & Yes & Yes & Yes \\
${ }^{[2]} \chi^{\alpha \beta} \gamma^{\mu \nu}{ }_{\rho}$ & Skewon-1 & No & Yes & Yes \\
${ }^{[3]} \chi^{\alpha \beta} \gamma^{\mu \nu}{ }_{\rho}$ & Skewon-2 & No & Yes & Yes \\
${ }^{[4]} \chi^{\alpha \beta} \gamma^{\mu \nu}{ }_{\rho}$ & Principal-2 & Yes & Yes & Yes \\
${ }^{[5]} \chi^{\alpha \beta} \gamma^{\mu \nu}{ }_{\rho}$ & Axion-1 & Yes & No & No \\
${ }^{[6]} \chi^{\alpha \beta} \gamma^{\mu \nu}{ }_{\rho}$ & Axion-2 & No & Yes & No \\
\hline
\end{tabular}

${ }^{+}{ }^{\alpha \beta}{ }_{\mu}{ }^{\gamma \delta}{ }_{\nu}={ }^{[1]} \chi^{\alpha \beta}{ }_{\mu}{ }^{\gamma \delta}{ }_{\nu}+{ }^{[4]} \chi^{\alpha \beta}{ }_{\mu}{ }^{\gamma \delta}{ }_{\nu}+{ }^{[5]} \chi^{\alpha \beta}{ }_{\mu}{ }^{\gamma \delta}{ }_{\nu}$,

$\bar{\chi}^{\alpha \beta}{ }_{\mu}{ }^{\gamma \delta}{ }_{\nu}={ }^{[2]} \chi^{\alpha \beta}{ }_{\mu}{ }^{\gamma \delta}{ }_{\nu}+{ }^{[3]} \chi^{\alpha \beta}{ }_{\mu}{ }^{\gamma \delta}{ }_{\nu}+{ }^{[6]} \chi^{\alpha \beta}{ }_{\mu}{ }^{\gamma \delta}{ }_{\nu}$.

We may call ${ }^{[1]} \chi$ a reversible symmetric principal part (principal-1), ${ }^{[4]} \chi$ a reversible antisymmetric principal part (principal-2), ${ }^{[5]} \chi$ a reversible axion (axion-1); likewise we may call ${ }^{[2]} \chi$ a skewon antisymmetric principal part (skewon-1), ${ }^{[3]} \chi$ a skewon symmetric principal part (skewon- 2 ), and ${ }^{[6]} \chi$ a skewon axion (axion-2). This is summarized in Table 1.

For completeness, we present here the results about the contributions of the irreducible pieces into the dispersion relation for the gravitational waves. These facts will be derived in Sect. 6.

\section{$3.3 S_{6}$ decomposition of $\check{\chi}_{\alpha \beta \mu \gamma \delta \nu}$}

Now we consider the decomposition of the constitutive tensor under the permutation of its six lower indices. We follow Hamermesh's [15] Eqs. (7-159) to (7-162). Then, for $n=4$ dimensions, the symmetries (35) imply the Young decomposition of $\check{\chi}_{\alpha \beta \mu \gamma \delta \nu}$ :

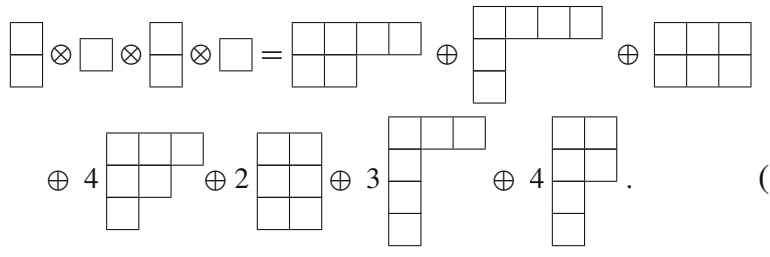

Here we omitted vanishing diagrams in $n=4$ (i.e. diagrams that contain antisymmetrization with respect to more than four indices). In terms of independent components, we have

$576=126 \oplus 70 \oplus 50 \oplus 4 \times 64 \oplus 2 \times 10$

$\oplus 3 \times 10 \oplus 4 \times 6$.

In tensorial language, this corresponds to

$\check{\chi}_{\alpha \beta \mu \gamma \delta \nu}=\sum_{I=1}^{7}{ }^{(I)} \mathbb{P}_{\alpha \beta \mu \gamma \kappa \delta \delta}^{\omega \lambda \kappa \sigma \epsilon} \check{\chi}_{\omega \lambda \kappa \rho \sigma \epsilon}=\sum_{I=1}^{7}{ }^{(I)} \check{\chi}_{\alpha \beta \mu \gamma \delta \nu}$, 
where again the expressions ${ }^{(I)} \mathbb{P}_{\alpha \beta \mu \gamma \delta \nu \nu}^{\omega \lambda \kappa \rho \epsilon}$ denote orthogonal projectors,

${ }^{(I)} \mathbb{P} \circ{ }^{(J)} \mathbb{P}={ }^{(I)} \mathbb{P} \delta^{I J}, \quad \delta^{I J}=\left\{\begin{array}{ll}1 & \text { for } I=J \\ 0 & \text { for } I \neq J\end{array}\right.$.

The explicit expressions of the seven terms ${ }^{(I)} \check{\chi}_{\alpha \beta \mu \gamma \delta v}$ are quite involved and we do not display them here; they can be found in "Appendix".

\subsection{Relation between the decompositions}

In order to relate the two inequivalent decompositions discussed above, it is useful to define

${ }^{\{I\}} \chi^{\alpha \beta}{ }_{\mu}{ }^{\gamma \delta}{ }_{\nu}:=\frac{1}{4} \epsilon^{\alpha \beta \omega \lambda} \epsilon^{\gamma \delta \rho \sigma(I)} \check{\chi}_{\omega \lambda \mu \rho \sigma \nu}$.

By using Eqs. (241)-(247), one may verify that the tensors ${ }^{\{I\}} \chi^{\alpha \beta}{ }_{\mu}^{\gamma \delta}{ }_{\nu}$ satisfy the relations

${ }^{\{I\}} \chi^{[\alpha \beta}{ }_{\mu}{ }^{\gamma \delta]}{ }_{\nu}=0$ for $I=1,2,3,4,5$,

${ }^{\{I\}} \chi_{[\mu}^{\alpha \beta}{ }_{\nu]}^{\gamma \delta}=0$ for $I=1,2$,

${ }^{\{I\}} \chi^{\alpha \beta}{ }_{\left(\mu^{\gamma \delta}\right.}{ }_{\nu)}=0$ for $I=3$,

as well as

${ }^{\{I\}} \chi^{\alpha \beta}{ }_{\mu}^{\gamma \delta}{ }_{v}=+{ }^{\{I\}} \chi^{\gamma \delta}{ }_{\mu}^{\alpha \beta}{ }_{v} \quad$ for $I=1,3$,

${ }^{\{I\}} \chi^{\alpha \beta}{ }_{\mu}{ }^{\gamma \delta}{ }_{\nu}=-{ }^{\{I\}} \chi^{\gamma \delta}{ }_{\mu}{ }^{\alpha \beta}{ }_{\nu}$ for $I=2$,

${ }^{\{I\}} \chi^{\alpha \beta}{ }_{\mu}{ }^{\gamma \delta}{ }_{\nu}=+{ }^{\{I\}} \chi^{\gamma \delta}{ }_{\nu}{ }^{\alpha \beta}{ }_{\mu}$ for $I=1,5$,

${ }^{\{I\}} \chi^{\alpha \beta}{ }_{\mu}{ }^{\gamma \delta}{ }_{\nu}=-{ }^{\{I\}} \chi^{\gamma \delta}{ }_{\nu}{ }^{\alpha \beta}{ }_{\mu}$ for $I=2,3$.

Eventually, we find

$$
\begin{aligned}
& { }^{\{6\}} \chi^{\alpha \beta}{ }_{\left[\mu^{\gamma \delta}\right.}{ }_{\nu]}+{ }^{\{6\}} \chi^{\gamma \delta}{ }_{[\mu}^{\alpha \beta}{ }_{\nu]}=0, \\
& { }^{\{6\}} \chi^{\alpha \beta}{ }_{(\mu}{ }^{\gamma \delta}{ }_{\nu)}+{ }^{\{6\}} \chi^{\gamma \delta}{ }_{(\mu}{ }^{\alpha \beta}{ }_{\nu)}={ }^{\{6\}} \chi^{[\alpha \beta}{ }_{(\mu}{ }^{\gamma \delta]}{ }_{\nu)} \text {, } \\
& { }^{\{7\}} \chi^{\alpha \beta}{ }_{(\mu}^{\gamma \delta}{ }_{v)}+{ }^{\{7\}} \chi^{\gamma \delta}{ }_{(\mu}^{\alpha \beta}{ }_{v)}=0 .
\end{aligned}
$$

We can apply directly the $S_{4} \times S_{2}$ decomposition to these order- $\left(\begin{array}{l}4 \\ 2\end{array}\right)$ tensors and compare the results to the tensors ${ }^{[I]} \chi^{\alpha \beta}{ }_{\mu}{ }^{\gamma \delta}{ }_{\nu}$. By extensive use of computer algebra, we find the following relations:

$$
\begin{aligned}
{ }^{[1]} \chi^{\alpha \beta}{ }_{\mu}^{\gamma \delta}{ }_{\nu}= & { }^{\{1\}} \chi^{\alpha \beta}{ }_{(\mu}{ }^{\gamma \delta}{ }_{\nu)}+{ }^{\{5\}} \chi^{\alpha \beta}{ }_{(\mu}{ }^{\gamma \delta}{ }_{\nu)} \\
& +\frac{1}{2}\left[{ }^{\{4\}} \chi^{\alpha \beta}{ }_{(\mu}{ }^{\gamma \delta}{ }_{\nu)}+{ }^{\{4\}} \chi^{\gamma \delta}{ }_{(\mu}^{\alpha \beta}{ }_{\nu)}\right], \\
{ }^{[2]} \chi^{\alpha \beta}{ }_{\mu}^{\gamma \delta}{ }_{\nu}= & { }^{\{3\}} \chi^{\alpha \beta}{ }_{[\mu}{ }^{\gamma \delta}{ }_{\nu]}-{ }^{\{7\}} \chi^{[\alpha \beta}{ }_{[\mu}{ }^{\gamma \delta]}{ }_{\nu]} \\
& +\frac{1}{2} \sum_{I=4,7}\left[{ }^{\{I\}} \chi^{\alpha \beta}{ }_{[\mu}^{\gamma \delta}{ }_{\nu]}+{ }^{\{I\}} \chi^{\gamma \delta}{ }_{[\mu}^{\alpha \beta}{ }_{\nu]}\right],
\end{aligned}
$$

$$
\begin{aligned}
& { }^{[3]} \chi^{\alpha \beta}{ }_{\mu}{ }^{\gamma \delta}{ }_{\nu}={ }^{\{2\}} \chi^{\alpha \beta}\left(\mu^{\gamma \delta}{ }_{\nu)}\right.
\end{aligned}
$$

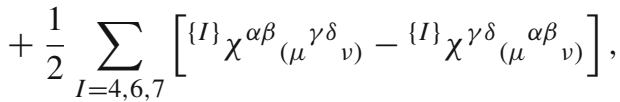

$$
\begin{aligned}
& { }^{[4]} \chi^{\alpha \beta}{ }_{\mu}{ }^{\gamma \delta}{ }_{\nu}={ }^{\{5\}} \chi^{\alpha \beta}{ }_{[\mu}{ }^{\gamma \delta}{ }_{\nu]} \\
& +\frac{1}{2} \sum_{I=4,6,7}\left[{ }^{\{I\}} \chi_{[\mu}^{\alpha \beta}{ }^{\gamma \delta}{ }_{\nu]}-{ }^{\{I\}} \chi^{\gamma \delta}{ }_{[\mu}^{\alpha \beta}{ }_{\nu]}\right],
\end{aligned}
$$

\subsection{Reversibility}

For reversible processes, as shown in (21), the constitutive tensor satisfies

$\chi^{\alpha \beta}{ }_{\mu}^{\gamma \delta}{ }_{\nu}=\chi_{\nu}^{\gamma \delta}{ }_{\nu}^{\alpha \beta}{ }_{\mu}$.

As we demonstrated above, the reversible and irreversible parts of the constitutive tensor are presented, respectively, as

$$
\begin{aligned}
& \stackrel{+}{\chi}^{\alpha \beta}{ }_{\mu}{ }^{\gamma \delta}{ }_{\nu}=\frac{1}{2}\left(\chi^{\alpha \beta}{ }_{\mu}{ }^{\gamma \delta}{ }_{\nu}+\chi^{\gamma \delta}{ }_{\nu}{ }^{\alpha \beta}{ }_{\mu}\right) \\
& ={ }^{[1]} \chi^{\alpha \beta}{ }_{\mu}^{\gamma \delta}{ }_{\nu}+{ }^{[4]} \chi^{\alpha \beta}{ }_{\mu}{ }^{\gamma \delta}{ }_{\nu}+{ }^{[5]} \chi^{\alpha \beta}{ }_{\mu}{ }^{\gamma \delta}{ }_{\nu} \text {, } \\
& \bar{\chi}^{\alpha \beta}{ }_{\mu}{ }^{\gamma \delta}{ }_{\nu}=\frac{1}{2}\left(\chi^{\alpha \beta}{ }_{\mu}{ }^{\gamma \delta}{ }_{\nu}-\chi^{\gamma \delta_{\nu} \alpha \beta}{ }_{\mu}\right) \\
& ={ }^{[2]} \chi^{\alpha \beta}{ }_{\mu}{ }^{\gamma \delta}{ }_{\nu}+{ }^{[3]} \chi^{\alpha \beta}{ }_{\mu}{ }^{\gamma \delta}{ }_{\nu}+{ }^{[6]} \chi^{\alpha \beta}{ }_{\mu}{ }^{\gamma \delta}{ }_{\nu} .
\end{aligned}
$$

This corresponds to $576=300 \oplus 276$, that is, the reversible constitutive tensor comprises 300 independent components. Employing relations Eqs. (75)-(80), one finds

$$
\begin{aligned}
& { }^{+}{ }^{\alpha \beta}{ }_{\mu}{ }^{\gamma \delta}{ }_{\nu}={ }^{\{1\}} \chi^{\alpha \beta}{ }_{(\mu}{ }^{\gamma \delta}{ }_{\nu)}+{ }^{\{5\}} \chi^{\alpha \beta}{ }_{\mu}{ }^{\gamma \delta}{ }_{\nu} \\
& +\frac{1}{2} \sum_{I=4,6,7}\left[{ }^{\{I\}} \chi^{\alpha \beta}{ }_{\mu}^{\gamma \delta}{ }_{\nu}+{ }^{\{I\}} \chi^{\gamma \delta}{ }_{\nu}^{\alpha \beta}{ }_{\mu}\right] \text {, } \\
& \bar{\chi}^{\alpha \beta}{ }_{\mu}{ }^{\gamma \delta}{ }_{\nu}={ }^{\{2\}} \chi^{\alpha \beta}\left(\mu^{\gamma \delta}{ }_{\nu)}+{ }^{\{3\}} \chi^{\alpha \beta}{ }_{[\mu}{ }^{\gamma \delta}{ }_{\nu]}\right. \\
& +\frac{1}{2} \sum_{I=4,6,7}\left[{ }^{\{I\}} \chi^{\alpha \beta} \mu_{\nu}^{\gamma \delta}-{ }^{\{I\}} \chi^{\gamma \delta}{ }_{\nu}^{\alpha \beta}{ }_{\mu}\right] \text {. }
\end{aligned}
$$

Let us emphasize that Eqs. (66) and (67) imply

$$
{ }^{\{1\}} \chi^{\alpha \beta}{ }_{\left[\mu^{\gamma \delta}{ }_{\nu]}\right.}=0,{ }^{\{2\}} \chi^{\alpha \beta}{ }_{\left[\mu^{\gamma \delta}{ }_{\nu]}\right.}=0,{ }^{\{3\}} \chi^{\alpha \beta}\left(\mu^{\gamma \delta}{ }_{\nu)}=0 .\right.
$$

In summary, we see that the reversible piece of the constitutive tensor $\chi^{\alpha \beta} \mu_{\mu}^{\gamma \delta}$ is comprised of the order- $\left(\begin{array}{l}0 \\ 6\end{array}\right)$ irreducible pieces $I=1,5$ as well as the appropriately (anti-) symmetrized parts of $I=4,6,7$. 


\subsection{Lagrangian}

Let us first consider how these independent pieces contribute to the gravitational Lagrangian ${ }^{(\vartheta)} \Lambda=-\frac{1}{2} F^{\alpha} \wedge H_{\alpha}$. In components, we have

${ }^{(\vartheta)} \Lambda=-\frac{1}{4} F_{\beta \gamma}{ }^{\alpha} \check{H}^{\beta \gamma}{ }_{\alpha}$ vol.

Substitution of the constitutive law (11) yields

${ }^{(\vartheta)} \Lambda=-\frac{1}{8} \chi^{\beta \gamma}{ }_{\alpha}^{\nu \rho}{ }_{\mu} F_{\beta \gamma}{ }^{\alpha} F_{\nu \rho}{ }^{\mu}$ vol.

Consequently only those parts of the constitutive tensor contribute to the Lagrangian that satisfy the "pair commutation" symmetries

$\chi^{\beta \gamma}{ }_{\alpha}^{\nu \rho}{ }_{\mu}=\chi^{\nu \rho}{ }_{\mu}^{\beta \gamma}{ }_{\alpha}$.

Accordingly, only the following terms are left over in the Lagrangian:

${ }^{(\vartheta)} \Lambda=-\frac{1}{8} \sum_{I=1,4,5}{ }^{[I]} \chi^{\beta \gamma}{ }_{\alpha}{ }^{\nu \rho}{ }_{\mu} F_{\beta \gamma}{ }^{\alpha} F_{\nu \rho}{ }^{\mu}$ vol.

\subsection{Parametrization of irreducible parts}

In premetric electrodynamics, there exists a convenient parametrization of the axion and skewon parts by a pseudo-scalar and a traceless second order tensor, see Sects. D.1.4 and D.1.5 of the book [22].

One may wish to develop a similar formalism for the gravitational case under consideration. By analogy with electrodynamics, let us introduce the following new objects:

$\oint_{\gamma \rho \sigma}{ }^{v}:=\frac{1}{4} \epsilon_{\alpha \beta \mu \sigma}{ }^{[3]} \chi^{\alpha \beta}{ }_{\gamma}^{\mu \nu}{ }_{\rho}$,

$Q_{\gamma \rho \sigma}{ }^{\nu}:=\frac{1}{4} \epsilon_{\alpha \beta \mu \sigma}{ }^{[4]} \chi^{\alpha \beta}{ }_{\gamma}^{\mu \nu}{ }_{\rho}$.

By construction (hint: use the definitions of ${ }^{[3]} \chi$ and ${ }^{[4]} \chi$ ), these tensors obey

$\$_{[\alpha \beta] \mu}{ }^{\nu}=0, \quad \$_{\alpha \beta \nu}{ }^{\nu}=0 ;$

$Q_{(\alpha \beta) \mu}{ }^{\nu}=0, \quad Q_{\alpha \beta \nu}{ }^{\nu}=0$.

Accordingly, the number of independent components for $\$_{\alpha \beta \mu}{ }^{\nu}$ is $10 \times 4 \times 4-10=150$ and for $Q_{\alpha \beta \mu}{ }^{\nu} 6 \times 4 \times 4-6=$ 90 , respectively. It is straightforward to see that

${ }^{[3]} \chi^{\alpha \beta} \gamma^{\mu \nu}{ }_{\rho}=\epsilon^{\alpha \beta \lambda[\mu} \phi_{\gamma \rho \lambda}{ }^{\nu]}-\epsilon^{\mu \nu \lambda[\alpha} \phi_{\gamma \rho \lambda}{ }^{\beta]}$,

${ }^{[4]} \chi^{\alpha \beta}{ }_{\gamma}{ }^{\mu \nu}{ }_{\rho}=\epsilon^{\alpha \beta \lambda[\mu} Q_{\gamma \rho \lambda}{ }^{\nu]}-\epsilon^{\mu \nu \lambda[\alpha} Q_{\gamma \rho \lambda}{ }^{\beta]}$.

Thereby, we found a complete parametrization of ${ }^{[3]} \chi$ and ${ }^{[4]} \chi$. This construction obviously generalizes the representation of a skewon in electrodynamics in terms of the traceless second order tensor [22]. In the context of the gravitational theory, however, only (95) refers to the skewon, whereas (96) parametrizes the reversible irreducible part.

Now, let us turn to the irreducible parts ${ }^{[3]} \chi$ and ${ }^{[4]} \chi$ for which we introduce

${ }^{[5]} A_{\gamma \rho}:=\frac{1}{4 !} \epsilon_{\alpha \beta \mu \nu}{ }^{[5]} \chi^{\alpha \beta} \gamma^{\mu \nu}{ }_{\rho}={ }^{[5]} A_{\rho \gamma}$,
${ }^{\left[{ }^{[6]} A_{\gamma \rho}\right.}:=\frac{1}{4 !} \epsilon_{\alpha \beta \mu \nu}{ }^{[6]} \chi^{\alpha \beta}{ }_{\gamma}{ }^{\mu \nu}{ }_{\rho}=-{ }^{\left[{ }^{[6]}\right.} A_{\rho \gamma}$.

The reciprocal relations read

${ }^{[5]} \chi^{\alpha \beta}{ }_{\gamma}^{\mu \nu}{ }_{\rho}={ }^{[5]} A_{\gamma \rho} \epsilon^{\alpha \beta \mu \nu}$,

${ }^{[6]} \chi^{\alpha \beta}{ }_{\gamma}{ }^{\mu \nu}{ }_{\rho}={ }^{[6]} A_{\gamma \rho} \epsilon^{\alpha \beta \mu \nu}$.

As a result, we find for the axion type part of the constitutive tensor

${ }^{\text {(ax) }} \chi^{\alpha \beta}{ }_{\gamma}^{\mu \nu}{ }_{\rho}=\mathcal{A}_{\gamma \rho} \epsilon^{\alpha \beta \mu \nu}, \quad \mathcal{A}_{\alpha \beta}:={ }^{[5]} A_{\alpha \beta}+{ }^{[6]} A_{\alpha \beta}$.

Summarizing, we demonstrated that the four irreducible parts ${ }^{[3]} \chi,{ }^{[4]} \chi,{ }^{[5]} \chi$, and ${ }^{[6]} \chi$ of the constitutive tensor can be conveniently parametrized, see Eqs. (95), (96), (99), and (100). They are expressed in terms of the tensors (91), (92), (97), and (98) with the correct number of independent components: for $\oint_{\alpha \beta \mu}{ }^{\nu} 150$, for $Q_{\alpha \beta \mu}{ }^{\nu} 90$, for ${ }^{[5]} A_{\alpha \beta} 10$, and for ${ }^{[6]} A_{\alpha \beta} 6$. The two remaining pieces ${ }^{[1]} \chi$ and ${ }^{[2]} \chi$ do not admit simple parametrizations, though.

\subsection{Energy-momentum current}

The energy-momentum current of the coframe field is defined as [31]

$\left.\left.{ }^{(\vartheta)} \Sigma_{\alpha}=\frac{1}{2}\left[F^{\beta} \wedge\left(e_{\alpha}\right\rfloor H_{\beta}\right)-H_{\beta} \wedge\left(e_{\alpha}\right\rfloor F^{\beta}\right)\right]$.

The constitutive law for the pure axion piece (101) is expressed as

${ }^{(\mathrm{ax})} H_{\alpha}=\mathcal{A}_{\alpha \beta} F^{\beta}$.

In this case, the energy-momentum current takes the form

$\left.{ }^{(\mathrm{ax})} \Sigma_{\alpha}=\mathcal{A}_{[\beta \gamma]} F^{\beta} \wedge\left(e_{\alpha}\right\rfloor F^{\gamma}\right)$.

Consequently, the symmetric combination of the axionic part ${ }^{[5]} \chi^{\beta \gamma}{ }_{\alpha}^{\nu \rho}{ }_{\mu}$ does not contribute to the energy-momentum current.

3.9 Contracting $\chi^{\alpha \beta} \gamma^{\mu \nu}{ }_{\rho}$ twice: four second order tensors

In electrodynamics, we can extract from the constitutive tensor $\chi^{\alpha \beta \gamma \delta}$ a pseudo-scalar density, the axion field, by contracting it with the Levi-Civita symbol: ${ }^{(\mathrm{ax})} \alpha:=\frac{1}{4 !} \varepsilon_{\alpha \beta \gamma \delta}$ 
$\chi^{\alpha \beta \gamma \delta}$. In gravity, we can only reduce the constitutive tensor $\chi^{\alpha \beta} \gamma_{\rho}^{\mu \nu}$ of type $\left(\begin{array}{l}4 \\ 2\end{array}\right)$ to four different 2 nd order tensors:

$$
\begin{aligned}
m^{\alpha \beta} & :=\chi^{\alpha \mu}{ }_{\mu}{ }^{\beta \nu}{ }_{\nu}, \\
n^{\alpha \beta} & :=\chi^{\alpha \mu}{ }_{\nu}{ }^{\beta \nu}{ }_{\mu}, \\
k^{\alpha \beta} & :=\chi^{\mu \nu}{ }_{\mu}^{\alpha \beta}{ }_{\nu}, \\
l^{\alpha \beta} & :=\chi^{\alpha \beta}{ }_{\mu}{ }^{\mu \nu}{ }_{v} .
\end{aligned}
$$

The construction of scalars is impossible at this premetric stage.

The tensors $k^{\alpha \beta}=-k^{\beta \alpha}$ and $l^{\alpha \beta}=-l^{\beta \alpha}$ are antisymmetric, that is, $k^{(\alpha \beta)}=0, l^{(\alpha \beta)}=0$, whereas $m^{\alpha \beta}$ and $n^{\alpha \beta}$ are asymmetric. However, in the reversible case, $m^{\alpha \beta}$ and $n^{\alpha \beta}$ turn out to be symmetric, whereas the two other antisymmetric tensors turn out to be transposed to each other, $k^{\alpha \beta}=l^{\beta \alpha}$. This can be recognized more clearly by collecting all the traces into the specific constitutive tensor

$$
\begin{aligned}
\chi_{\gamma}^{\alpha \beta}{ }^{\mu \nu}{ }_{\rho}= & \mathcal{L}^{\alpha \beta} \delta_{\gamma}^{[\mu} \delta_{\rho}^{\nu]}+\mathcal{K}^{\mu \nu} \delta_{\rho}^{[\alpha} \delta_{\gamma}^{\beta]} \\
& +\delta_{\gamma}^{[\alpha} \mathcal{M}^{\beta][\mu} \delta_{\rho}^{\nu]}+\delta_{\rho}^{[\alpha} \mathcal{N}^{\beta][\mu} \delta_{\gamma}^{\nu]} .
\end{aligned}
$$

This tensor is determined by four tensors of 2 nd order: two of them are antisymmetric, $\mathcal{L}^{\alpha \beta}=-\mathcal{L}^{\beta \alpha}$ and $\mathcal{K}^{\alpha \beta}=-\mathcal{K}^{\beta \alpha}$, that is, $\mathcal{L}^{(\alpha \beta)}=0, \mathcal{K}^{(\alpha \beta)}=0$, and the two other ones are general 2nd order tensors $\mathcal{M}^{\alpha \beta}$ and $\mathcal{N}^{\alpha \beta}$.

It is straightforward to establish one-to-one correspondences between these objects and the traces (105)-(108):

$$
\begin{aligned}
m^{\alpha \beta}= & \frac{3}{2}\left(\mathcal{L}^{\alpha \beta}-\mathcal{K}^{\alpha \beta}\right) \\
& -\frac{1}{4}\left(9 \mathcal{M}^{\alpha \beta}+2 \mathcal{N}^{\alpha \beta}+\mathcal{N}^{\beta \alpha}\right), \\
n^{\alpha \beta}= & \frac{3}{2}\left(\mathcal{K}^{\alpha \beta}-\mathcal{L}^{\alpha \beta}\right) \\
& -\frac{1}{4}\left(9 \mathcal{N}^{\alpha \beta}+2 \mathcal{M}^{\alpha \beta}+\mathcal{M}^{\beta \alpha}\right), \\
k^{\alpha \beta}= & \mathcal{L}^{\alpha \beta}-6 \mathcal{K}^{\alpha \beta}-\frac{3}{2} \mathcal{M}^{[\alpha \beta]}+\frac{3}{2} \mathcal{N}^{[\alpha \beta]}, \\
l^{\alpha \beta}= & 6 \mathcal{L}^{\alpha \beta}-\mathcal{K}^{\alpha \beta}-\frac{3}{2} \mathcal{M}^{[\alpha \beta]}+\frac{3}{2} \mathcal{N}^{[\alpha \beta]} .
\end{aligned}
$$

The inverse relations read

$$
\begin{aligned}
\mathcal{L}^{\alpha \beta}= & \frac{1}{10}\left(3 l^{\alpha \beta}+k^{\alpha \beta}-3 m^{[\alpha \beta]}+3 n^{[\alpha \beta]}\right), \\
\mathcal{K}^{\alpha \beta}= & \frac{1}{10}\left(-l^{\alpha \beta}-3 k^{\alpha \beta}+3 m^{[\alpha \beta]}-3 n^{[\alpha \beta]}\right), \\
\mathcal{M}^{\alpha \beta}= & \frac{1}{10}\left(3 l^{\alpha \beta}+3 k^{\alpha \beta}-9 m^{[\alpha \beta]}+5 n^{[\alpha \beta]}\right) \\
& -\frac{1}{6}\left(3 m^{(\alpha \beta)}-n^{(\alpha \beta)}\right), \\
\mathcal{N}^{\alpha \beta}= & \frac{1}{10}\left(-3 l^{\alpha \beta}-3 k^{\alpha \beta}+5 m^{[\alpha \beta]}-9 n^{[\alpha \beta]}\right) \\
& +\frac{1}{6}\left(m^{(\alpha \beta)}-3 n^{(\alpha \beta)}\right) .
\end{aligned}
$$

Decomposing the specific constitutive tensor (109) into reversible and irreversible (skewon) parts yields

$$
\begin{aligned}
\stackrel{ \pm}{\chi}^{\alpha \beta}{ }_{\gamma}^{\mu \nu}{ }_{\rho}= & \stackrel{ \pm}{\mathcal{L}^{\alpha \beta}} \delta_{\gamma}^{[\mu} \delta_{\rho}^{\nu]} \pm \stackrel{ \pm}{\mathcal{L}^{\mu \nu}} \delta_{\rho}^{[\alpha} \delta_{\gamma}^{\beta]} \\
& +\delta_{\gamma}^{[\alpha} \stackrel{ \pm}{\mathcal{M}^{\beta]}}{ }^{\beta]} \delta_{\rho}^{\nu]}+\delta_{\rho}^{[\alpha} \stackrel{\mathcal{N}}{\mathcal{N}}^{\beta][\mu} \delta_{\gamma}^{\nu]} .
\end{aligned}
$$

Here we introduced

$$
\begin{aligned}
\stackrel{\mathcal{L}}{ }^{\alpha \beta} & =\frac{1}{2}\left(\mathcal{L}^{\alpha \beta} \pm \mathcal{K}^{\alpha \beta}\right), \\
\stackrel{\mathcal{M}}{ }^{\alpha \beta} & =\mathcal{M}^{(\alpha \beta)}, \quad \overline{\mathcal{M}}^{\alpha \beta}=\mathcal{M}^{[\alpha \beta]}, \\
\stackrel{+}{\mathcal{N}}^{\alpha \beta} & =\mathcal{N}^{(\alpha \beta)}, \quad \overline{\mathcal{N}}^{\alpha \beta}=\mathcal{N}^{[\alpha \beta]} .
\end{aligned}
$$

This confirms that the irreversible, the skewon part vanishes, $\bar{\chi}^{\alpha \beta}{ }_{\gamma}{ }^{\mu \nu}{ }_{\rho}=0$, provided $\overline{\mathcal{L}}^{\alpha \beta}=0, \overline{\mathcal{M}}^{\alpha \beta}=0$, and $\overline{\mathcal{N}}^{\alpha \beta}=0$, i.e., when $m^{\alpha \beta}$ and $n^{\alpha \beta}$ are symmetric and $k^{\alpha \beta}=l^{\beta \alpha}$.

In the general case, the specific constitutive trace tensor (109) is characterized by 5 nontrivial irreducible parts:

$$
\begin{aligned}
& { }^{[1]} \chi^{\alpha \beta}{ }_{\gamma}{ }^{\mu \nu}{ }_{\rho}=\delta_{(\gamma}^{[\alpha} \stackrel{S}{\mathcal{R}}^{\beta][\mu} \delta_{\rho)}^{\nu]}, \\
& { }^{[2]} \chi^{\alpha \beta}{ }_{\gamma}{ }^{\mu \nu}{ }_{\rho}=\overline{\mathcal{L}}^{\alpha \beta} \delta_{\gamma}^{[\mu} \delta_{\rho}^{\nu]}-\overline{\mathcal{L}}^{\mu v} \delta_{\rho}^{[\alpha} \delta_{\gamma}^{\beta]} \\
& +\delta_{[\gamma}^{[\alpha} \stackrel{a}{\mathcal{S}}^{\beta][\mu} \delta_{\rho]}^{\nu]}-\mathcal{A}^{[\alpha \beta} \delta_{\gamma}^{\mu} \delta_{\rho}^{\nu]}, \\
& { }^{[3]} \chi^{\alpha \beta}{ }_{\gamma}{ }^{\mu \nu}{ }_{\rho}=\delta_{(\gamma}^{[\alpha} \mathcal{S}^{\beta][\mu} \delta_{\rho)}^{\nu]}, \\
& { }^{[4]} \chi^{\alpha \beta}{ }^{\mu \nu}{ }_{\rho}{ }_{\rho}=\stackrel{+}{\mathcal{L}}^{\alpha \beta} \delta_{\gamma}^{[\mu} \delta_{\rho}^{\nu]}+\stackrel{+}{\mathcal{L}}^{\mu \nu} \delta_{\rho}^{[\alpha} \delta_{\gamma}^{\beta]} \\
& \left.+\delta_{[\gamma}^{[\alpha} \stackrel{a}{\mathcal{R}} \beta\right]\left[\mu \delta_{\rho]}^{\nu]},\right. \\
& { }^{[5]} \chi^{\alpha \beta}{ }_{\gamma}{ }^{\mu \nu}{ }_{\rho}=0 \text {, } \\
& { }^{[6]} \chi^{\alpha \beta}{ }^{\mu \nu}{ }_{\rho}=\mathcal{A}^{[\alpha \beta} \delta_{\gamma}^{\mu} \delta_{\rho}^{\nu]} \text {. }
\end{aligned}
$$

Here we denoted

$$
\begin{aligned}
& \mathcal{A}^{\alpha \beta}:=2 \overline{\mathcal{L}}^{\alpha \beta}+\overline{\mathcal{M}}^{\alpha \beta}-\overline{\mathcal{N}}^{\alpha \beta}, \\
& \stackrel{s}{\mathcal{R}}^{\alpha \beta}:=\stackrel{+}{\mathcal{M}}^{\alpha \beta}+\stackrel{+}{\mathcal{N}}^{\alpha \beta}, \\
& \stackrel{a}{\mathcal{R}}^{\alpha \beta}:=\stackrel{+}{\mathcal{M}}^{\alpha \beta}-\stackrel{+}{\mathcal{N}}^{\alpha \beta}, \\
& \stackrel{s}{\mathcal{S}}^{\alpha \beta}:=\overline{\mathcal{M}}^{\alpha \beta}+\overline{\mathcal{N}}^{\alpha \beta}, \\
& \stackrel{a}{\mathcal{S}}^{\alpha \beta}:=\overline{\mathcal{M}}^{\alpha \beta}-\overline{\mathcal{N}}^{\alpha \beta} \text {. }
\end{aligned}
$$

The first object (128) represents a skewonic axion, whereas the four other objects (129)-(132) describe contributions to the principal reversible and irreversible (skewon) parts. In the reversible case, only the first irreducible piece of Eq. (122) survives and the fourth one of Eq. (125). 


\section{Metric-dependent constitutive tensor}

In general relativity as well as in teleparallelism, the existence of a metric tensor $g$ is conventionally assumed. We choose the metric signature $(+,-,-,-)$. The question is then how the constitutive tensor density $\chi^{\alpha \beta}{ }_{\mu}{ }^{\gamma \delta}{ }_{\nu}$ can be expressed in terms of the metric, namely in terms of its covariant and/or contravariant components $g_{\alpha \beta}$ and $g^{\gamma \delta}$, respectively. Since $\chi$ is a 6th order tensor of type $\left(\begin{array}{l}4 \\ 2\end{array}\right)$, it seems reasonable to start with an ansatz of a purely contravariant dimensionless 6th order tensor $K^{\alpha \beta \mu \gamma \delta \nu}$. Then the contravariant metric components $g^{\gamma \delta}$ are the only metric components that have to be taken into account. In general, one can come up with a polynomial ansatz of arbitrary order in the metric tensor. However, since the resulting constitutive tensor is of 6th order, all indices of the metric factors except six should be necessarily contracted. Recalling that a contraction of the covariant and contravariant metric yields a Kronecker delta, we then find that a polynomial of an arbitrary order is automatically reduced to a general polynomial expression in the metric which is just cubic in $g^{\alpha \beta}$.

\subsection{A metric-dependent ansatz with parity conserving terms}

If we look only for parity conserving (even) pieces in $\chi$, the totally antisymmetric unit tensor is not allowed in the polynomial expression. Thus, for the 6th order tensor $K^{\alpha \beta \mu \gamma \delta \nu}$, the most general cubic expression in the metric, omitting terms explicitly symmetric in the index pairs $\alpha \beta$ and $\gamma \delta$, reads

$$
\begin{aligned}
K^{\alpha \beta \mu \gamma \delta \nu}= & \alpha_{1} g^{\alpha \mu} g^{\beta \gamma} g^{\delta \nu}+\alpha_{2} g^{\alpha \mu} g^{\beta \delta} g^{\gamma \nu}+\alpha_{3} g^{\alpha \gamma} g^{\beta \mu} g^{\delta v} \\
& +\alpha_{4} g^{\alpha \gamma} g^{\beta \delta} g^{\mu \nu}+\alpha_{5} g^{\alpha \gamma} g^{\beta \nu} g^{\mu \delta}+\alpha_{6} g^{\alpha \delta} g^{\beta \mu} g^{\gamma \nu} \\
& +\alpha_{7} g^{\alpha \delta} g^{\beta \gamma} g^{\mu \nu}+\alpha_{8} g^{\alpha \delta} g^{\beta \nu} g^{\gamma \mu}+\alpha_{9} g^{\alpha \nu} g^{\beta \gamma} g^{\mu \delta} \\
& +\alpha_{10} g^{\alpha \nu} g^{\beta \delta} g^{\mu \nu},
\end{aligned}
$$

where $\alpha_{1}, \ldots, \alpha_{10}$ are constants.

In order to tailor the ansatz (133) for our constitutive tensor $\chi^{\alpha \beta}{ }_{\mu}^{\gamma \delta}{ }_{\nu}$, we lower the indices $\mu$ and $\nu$ and, at the same time, we reorder terms with only one metric tensor such that the metric tensor is sandwiched in between two Kronecker deltas:

$$
\begin{aligned}
K_{\mu}^{\alpha \beta}{ }_{\mu}^{\gamma \delta}{ }_{\nu}= & \alpha_{1} \delta_{\mu}^{\alpha} g^{\beta \gamma} \delta_{v}^{\delta}+\alpha_{2} \delta_{\mu}^{\alpha} g^{\beta \delta} \delta_{v}^{\gamma}+\alpha_{3} \delta_{\mu}^{\beta} g^{\alpha \gamma} \delta_{v}^{\delta} \\
& +\alpha_{4} g^{\alpha \gamma} g^{\beta \delta} g_{\mu \nu}+\alpha_{5} \delta_{\nu}^{\beta} g^{\alpha \gamma} \delta_{\mu}^{\delta}+\alpha_{6} \delta_{\mu}^{\beta} g^{\alpha \delta} \delta_{v}^{\gamma} \\
& +\alpha_{7} g^{\alpha \delta} g^{\beta \gamma} g_{\mu \nu}+\alpha_{8} \delta_{\nu}^{\beta} g^{\alpha \delta} \delta_{\mu}^{\gamma}+\alpha_{9} \delta_{\nu}^{\alpha} g^{\beta \gamma} \delta_{\mu}^{\delta} \\
& +\alpha_{10} \delta_{\nu}^{\alpha} g^{\beta \delta} \delta_{\mu}^{\gamma} .
\end{aligned}
$$

Since $\chi^{\alpha \beta}{ }_{\mu}{ }^{\gamma \delta}{ }_{\nu}$ is antisymmetric in the index pairs $\alpha \beta$ and $\gamma \delta$, we have to antisymmetrize $K$ correspondingly by subsequently putting brackets around the indices $\alpha \beta$ and $\gamma \delta$, respectively. Also recall that the metric is symmetric, hence its index ordering is optional:

$$
\begin{aligned}
& K^{[\alpha \beta]}{ }_{\mu}^{[\gamma \delta]}{ }_{\nu} \\
&= \alpha_{1} \delta_{\mu}^{[\alpha} g^{\beta][\gamma} \delta_{\nu}^{\delta]}+\alpha_{2} \delta_{\mu}^{[\alpha} g^{\beta][\delta} \delta_{\nu}^{\gamma]}+\alpha_{3} \delta_{\mu}^{[\beta} g^{\alpha][\gamma} \delta_{\nu}^{\delta]} \\
&+\alpha_{4} g^{\gamma[\alpha} g^{\beta] \delta} g_{\mu \nu}+\alpha_{5} \delta_{\nu}^{[\beta} g^{\alpha][\gamma} \delta_{\mu}^{\delta]}+\alpha_{6} \delta_{\mu}^{[\beta} g^{\alpha][\delta} \delta_{\nu}^{\gamma]} \\
&+\alpha_{7} g^{\delta[\alpha} g^{\beta] \gamma} g_{\mu \nu}+\alpha_{8} \delta_{\nu}^{[\beta} g^{\alpha][\delta} \delta_{\mu}^{\gamma]}+\alpha_{9} \delta_{\nu}^{[\alpha} g^{\beta][\gamma} \delta_{\mu}^{\delta]} \\
&+\alpha_{10} \delta_{\nu}^{[\alpha} g^{\beta][\delta} \delta_{\mu}^{\gamma]} .
\end{aligned}
$$

Note that the $\alpha_{4}$ and the $\alpha_{7}$ terms are already antisymmetric in $\gamma \delta$. Thus, there is no need to put brackets [ ] around $\gamma \delta$ or $\delta \gamma$, respectively. We can now collect all terms by recalling the antisymmetry in both index pairs $\alpha \beta$ and $\gamma \delta$. We find, without any further intermediary step, the compact equation

$$
\begin{aligned}
K_{\mu}^{[\alpha \beta]}{ }_{\mu}^{[\gamma \delta]}{ }_{\nu} & =\left(\alpha_{4}-\alpha_{7}\right) g^{\gamma[\alpha} g^{\beta] \delta} g_{\mu \nu} \\
& +\left(\alpha_{1}-\alpha_{2}-\alpha_{3}+\alpha_{6}\right) \delta_{\mu}^{[\alpha} g^{\beta][\gamma} \delta_{\nu}^{\delta]} \\
& +\left(-\alpha_{5}+\alpha_{8}+\alpha_{9}-\alpha_{10}\right) \delta_{\nu}^{[\alpha} g^{\beta][\gamma} \delta_{\mu}^{\delta]} .
\end{aligned}
$$

We know that $\chi$ is a density, like $\sqrt{-g}$, with $g:=\operatorname{det} g_{\mu \nu}$. Accordingly, we can identify the parity even part of the constitutive tensor density as follows,

${ }^{\text {even }} \chi_{\mu}^{\alpha \beta}{ }_{\nu}^{\gamma \delta}(g)=\frac{\sqrt{-g}}{\varkappa} K_{\mu}^{[\alpha \beta]}{ }_{\nu}^{[\gamma \delta]}$,

with $\varkappa$ as the gravitational constant. In our formalism up to now, including the Tonti diagram, $\varkappa$ was put to 1 for simplicity. Here we do display it for clarity. Thus, we find, with the three constants,

$$
\left.\begin{array}{l}
\beta_{1}:=\alpha_{4}-\alpha_{7} \\
\beta_{2}:=\alpha_{1}-\alpha_{2}-\alpha_{3}+\alpha_{6} \\
\beta_{3}:=-\alpha_{5}+\alpha_{8}+\alpha_{9}-\alpha_{10},
\end{array}\right\}
$$

the expression

$$
\begin{aligned}
{ }^{\text {even }} \chi^{\alpha \beta}{ }_{\mu}{ }_{\nu}{ }_{\nu}(g) & =\frac{\sqrt{-g}}{\varkappa}\left(\beta_{1} g^{\gamma[\alpha} g^{\beta] \delta} g_{\mu \nu}\right. \\
& \left.+\beta_{2} \delta_{\mu}^{[\alpha} g^{\beta][\gamma} \delta_{\nu}^{\delta]}+\beta_{3} \delta_{\nu}^{[\alpha} g^{\beta][\gamma} \delta_{\mu}^{\delta]}\right) .
\end{aligned}
$$

This proves our ansatz with three independent constants in [31, Eq. (80)]..$^{5}$ It is, indeed, the most general expression which, up to a factor of $\sqrt{-g}$, turns out to be a cubic polynomial in the metric.

\footnotetext{
5 In [31], there was a typo in Eq. (80): the last plus sign + in this formula should have been a minus sign - Moreover, for simplification, we changed now our conventions with respect to the $\beta$ 's slightly: $4 \beta_{1}^{\text {old }}=$ $\beta_{1}, 8 \beta_{2}^{\text {old }}=-\beta_{2}, 8 \beta_{3}^{\text {old }}=-\beta_{3}$.
} 


\subsection{Parity violating terms}

Already for quite some time, see Pellegrini and Plebanski [48] and Müller-Hoissen and Nitsch [40], also parity violating (odd) constitutive tensors for torsion square Lagrangians have been considered in the literature; for recent reviews, see $[3,7,32,45]$. There it has been shown that two parity odd terms occur, which are both linear in the totally antisymmetric Levi-Civita symbol $\epsilon^{\alpha \beta \gamma \delta}$, which is, as we may recall, a tensor density:

${ }^{\text {odd }} \chi^{\alpha \beta}{ }_{\mu}^{\gamma \delta}{ }_{\nu}(g)=\frac{1}{\varkappa}\left\{\beta_{4} \epsilon^{\alpha \beta \gamma \delta} g_{\mu \nu}+\beta_{5} \epsilon^{\alpha \beta[\gamma}{ }_{[\mu} \delta_{\nu]}^{\delta]}\right\}$.

One can also take into account such additional parity violating terms in the framework of the Poincaré gauge theory of gravity. In that more general theory, it leads to interesting new cosmological models $[4,25]$ and to new gravitational wave solutions $[6,43]$.

We would like to understand the generality of the result in (140) in a similar way as we did it for the parity even case. For parity reasons, the Levi-Civita symbol has to be linearly or in odd powers in the corresponding ansatz. Since again, as in the even part of $\chi^{\alpha \beta}{ }_{\mu}^{\gamma \delta}{ }_{\nu}$, we start with an tensor expression of type $\left(\begin{array}{l}6 \\ 0\end{array}\right)$, we need additionally a tensor of type $\left(\begin{array}{l}2 \\ 0\end{array}\right)$. Clearly the contravariant components of the metric $g^{\alpha \beta}$ qualify for such a purpose. Thus, the simplest possible ansatz, see also (140), is to start with a 6th order tensor density as follows:

$$
\begin{aligned}
M^{\alpha \beta \mu \gamma \delta v}= & \mu_{1} g^{\alpha \mu} \varepsilon^{\beta \gamma \delta v}+\mu_{2} g^{\alpha \gamma} \varepsilon^{\beta \mu \delta v}+\mu_{3} g^{\alpha \delta} \varepsilon^{\beta \mu \gamma v} \\
& +\mu_{4} g^{\alpha \nu} \varepsilon^{\beta \mu \gamma \delta}+\mu_{5} g^{\beta \mu} \varepsilon^{\alpha \gamma \delta v}+\mu_{6} g^{\beta \gamma} \varepsilon^{\alpha \mu \delta v} \\
& +\mu_{7} g^{\beta \delta} \varepsilon^{\alpha \mu \gamma v}+\mu_{8} g^{\beta v} \varepsilon^{\alpha \mu \gamma \delta}+\mu_{9} g^{\mu \gamma} \varepsilon^{\alpha \beta \delta v} \\
& +\mu_{10} g^{\mu \delta} \varepsilon^{\alpha \beta \gamma v}+\mu_{11} g^{\mu v} \varepsilon^{\alpha \beta \gamma \delta}+\mu_{12} g^{\gamma v} \varepsilon^{\alpha \beta \mu \delta} \\
& +\mu_{13} g^{\delta v} \varepsilon^{\alpha \beta \mu \gamma} .
\end{aligned}
$$

The $\mu_{1}, \mu_{2}, \ldots, \mu_{13}$ are arbitrary constants. We now lower again the indices $\mu$ and $v$ and antisymmetrize at the same time with respect to the index pairs $\alpha \beta$ and $\gamma \delta$, respectively:

$$
\begin{aligned}
& M_{\mu}^{[\alpha \beta]}{ }_{\mu}^{[\gamma \delta]}{ }_{\nu}=\mu_{11} g_{\mu \nu} \varepsilon^{\alpha \beta \gamma \delta} \\
& \quad+\left(\mu_{1}-\mu_{5}\right) \delta_{\mu}^{[\alpha} \varepsilon^{\beta] \gamma \delta}{ }_{\nu}+\left(\mu_{4}-\mu_{8}\right) \delta_{\nu}^{[\alpha} \varepsilon^{\beta] \gamma \delta}{ }_{\mu} \\
& +\left(\mu_{9}-\mu_{10}\right) \delta_{\mu}^{[\gamma} \varepsilon^{\delta] \alpha \beta}{ }_{\nu}-\left(\mu_{12}-\mu_{13}\right) \delta_{\nu}^{[\gamma} \varepsilon_{\mu}^{\delta] \alpha \beta}{ }_{\mu} \\
& +\frac{1}{2}\left(-\mu_{2}+\mu_{3}+\mu_{6}-\mu_{7}\right)\left(g^{\gamma[\alpha} \varepsilon^{\beta] \delta}{ }_{\mu \nu}-g^{\delta[\alpha} \varepsilon^{\beta] \gamma}{ }_{\mu \nu}\right) .
\end{aligned}
$$

Having established the required antisymmetries, we can now identify the odd piece of the constitutive tensor as

$$
{ }^{\text {odd }} \chi^{\alpha \beta}{ }_{\mu}^{\gamma \delta}{ }_{\nu}(g)=\frac{1}{\varkappa} M_{\mu}^{[\alpha \beta]}{ }_{\nu}^{[\gamma \delta]}{ }_{\nu}
$$

Here we have to recall an algebraic trick that we had already applied in discussing the electromagnetic 4th order constitutive tensor, see [22, Eq. (D.1.65)]. Since in four dimensions the indices have always four values, any expression antisymmetrized over five indices vanishes identically. Thus, for an arbitrary tensor $T_{\cdots}^{\alpha \beta \gamma \delta \varepsilon \cdots}$, we have $T_{\ldots}^{[\alpha \beta \gamma \delta \varepsilon] \cdots} \equiv$ 0 . Accordingly, we have, for example, $T^{[\rho} \varepsilon^{\alpha \beta \gamma \delta]}=0$ or, if we evaluate the brackets,

$T^{\rho} \varepsilon^{\alpha \beta \gamma \delta} \equiv T^{\alpha} \varepsilon^{\rho \beta \gamma \delta}+T^{\beta} \varepsilon^{\alpha \rho \gamma \delta}+T^{\gamma} \varepsilon^{\alpha \beta \rho \delta}+T^{\delta} \varepsilon^{\alpha \beta \gamma \rho}$.

Looking now at (142), we have typically $\delta_{\mu}^{[\alpha} \varepsilon^{\beta] \gamma \delta \nu}$. With $T^{\alpha}{ }_{\mu}=\delta_{\mu}^{\alpha}$, we find

$\delta_{\mu}^{[\alpha} \varepsilon^{\beta] \gamma \delta v} \equiv \delta_{\mu}^{[\beta} \varepsilon^{\alpha] \gamma \delta v}+\delta_{\mu}^{\gamma} \varepsilon^{\beta \alpha \delta v}+\delta_{\mu}^{\delta} \varepsilon^{\beta \gamma \alpha \nu}+\delta_{\mu}^{\nu} \varepsilon^{\beta \gamma \delta \alpha}$.

By such methods, we can derive several identities for the Levi-Civita symbol in 4 dimensions:

$\begin{aligned} \varepsilon^{\alpha \beta[\gamma}{ }_{(\mu} \delta_{\nu)}^{\delta]}+\varepsilon^{\gamma \delta[\alpha}{ }_{(\mu} \delta_{\nu)}^{\beta]} & \equiv \frac{1}{2} \varepsilon^{\alpha \beta \gamma \delta} g_{\mu \nu}, \\ \varepsilon^{\alpha \beta[\gamma}{ }_{[\mu} \delta_{\nu]}^{\delta]}+\varepsilon^{\gamma \delta[\alpha}{ }_{[\mu} \delta_{\nu]}^{\beta]} & \equiv 0, \\ g^{\gamma[\alpha} \varepsilon^{\beta] \delta}{ }_{\mu \nu}-g^{\delta[\alpha} \varepsilon^{\beta] \gamma}{ }_{\mu \nu} & \equiv 2 \varepsilon^{\alpha \beta[\gamma}{ }_{[\mu} \delta_{\nu]}^{\delta]} .\end{aligned}$

Let us come back to (143) with (142). We decompose the $\delta \varepsilon$-terms in symmetric and antisymmetric pieces with respect to the lower indices:

$\delta_{\mu}^{[\alpha} \varepsilon^{\beta] \gamma \delta}{ }_{\nu}=\delta_{(\mu}^{[\alpha} \varepsilon^{\beta] \gamma \delta}{ }_{\nu)}+\delta_{[\mu}^{[\alpha} \varepsilon^{\beta] \gamma \delta}{ }_{\nu]}$,

$\delta_{\mu}^{[\gamma} \varepsilon^{\delta] \alpha \beta}{ }_{\nu}=\delta_{(\mu}^{[\gamma} \varepsilon^{\delta] \alpha \beta}{ }_{\nu)}+\delta_{[\mu}^{[\gamma} \varepsilon^{\delta] \alpha \beta}{ }_{\nu]}$.

As a result, the $\delta \varepsilon$-terms in the second and third lines of (142) can be rearranged as follows:

$$
\begin{aligned}
& \left(\mu_{1}-\mu_{5}+\mu_{4}-\mu_{8}\right) \delta_{(\mu}^{[\alpha} \varepsilon^{\beta] \gamma \delta}{ }_{\nu)} \\
& +\left(\mu_{9}-\mu_{10}-\mu_{12}+\mu_{13}\right) \delta_{(\mu}^{[\gamma} \varepsilon^{\delta] \alpha \beta}{ }_{\nu)} \\
& +\left(\mu_{1}-\mu_{5}-\mu_{4}+\mu_{8}\right) \delta_{[\mu}^{[\alpha} \varepsilon^{\beta] \gamma \delta}{ }_{\nu]} \\
& +\left(\mu_{9}-\mu_{10}+\mu_{12}-\mu_{13}\right) \delta_{[\mu}^{[\gamma} \varepsilon^{\delta] \alpha \beta}{ }_{\nu]} .
\end{aligned}
$$

Next, we write

$$
\begin{aligned}
\delta_{(\mu}^{[\alpha} \varepsilon^{\beta] \gamma \delta}{ }_{\nu)} & \frac{1}{2}\left(\delta_{(\mu}^{[\alpha} \varepsilon^{\beta] \gamma \delta}{ }_{\nu)}+\delta_{(\mu}^{[\gamma} \varepsilon^{\delta] \alpha \beta}{ }_{\nu)}\right) \\
& +\frac{1}{2}\left(\delta_{(\mu}^{[\alpha} \varepsilon^{\beta] \gamma \delta}{ }_{\nu)}-\delta_{(\mu}^{[\gamma} \varepsilon^{\delta] \alpha \beta}{ }_{\nu)}\right), \\
\delta_{(\mu}^{[\gamma} \varepsilon^{\delta] \alpha \beta}{ }_{\nu)}= & \frac{1}{2}\left(\delta_{(\mu}^{[\gamma} \varepsilon^{\delta] \alpha \beta}{ }_{\nu)}+\delta_{(\mu}^{[\alpha} \varepsilon^{\beta] \gamma \delta_{\nu)}}\right) \\
& +\frac{1}{2}\left(\delta_{(\mu}^{[\gamma} \varepsilon^{\delta] \alpha \beta}{ }_{\nu)}-\delta_{(\mu}^{[\alpha} \varepsilon^{\beta] \gamma \delta_{\nu)}}\right) .
\end{aligned}
$$


Consequently, the expression (151) transforms into

$$
\begin{aligned}
& \left(\mu_{1}-\mu_{5}-\mu_{4}+\mu_{8}-\mu_{9}+\mu_{10}-\mu_{12}+\mu_{13}\right) \delta_{[\mu}^{[\alpha} \varepsilon^{\beta] \gamma \delta}{ }_{\nu]} \\
& +\frac{1}{2}\left(\mu_{1}-\mu_{5}+\mu_{4}-\mu_{8}-\mu_{9}+\mu_{10}+\mu_{12}-\mu_{13}\right) \\
& \times\left(\delta_{(\mu}^{[\alpha} \varepsilon^{\beta] \gamma \delta}{ }_{\nu)}-\delta_{(\mu}^{[\gamma} \varepsilon^{\delta] \alpha \beta}{ }_{\nu)}\right) \\
& +\frac{1}{2}\left(\mu_{1}-\mu_{5}+\mu_{4}-\mu_{8}+\mu_{9}-\mu_{10}-\mu_{12}+\mu_{13}\right) \\
& \times\left(\delta_{(\mu}^{[\alpha} \varepsilon^{\beta] \gamma \delta}{ }_{\nu)}+\delta_{(\mu}^{[\gamma} \varepsilon^{\delta] \alpha \beta}{ }_{\nu)}\right) .
\end{aligned}
$$

where we used the identity (147). We recall that (154) belongs to the second and third line of (142).

In the last step, it remains to use (146) in the last line of (154) and to substitute (148) into the last line of (142). Then, with the constants

$$
\begin{aligned}
\beta_{4}:= & \frac{1}{4}\left(\mu_{1}-\mu_{5}+\mu_{4}-\mu_{8}+\mu_{9}-\mu_{10}-\mu_{12}+\mu_{13}+4 \mu_{11}\right), \\
\beta_{5}:= & \mu_{1}-\mu_{5}-\mu_{4}+\mu_{8}-\mu_{9}+\mu_{10} \\
& +\mu_{12}-\mu_{13}+\mu_{2}-\mu_{6}-\mu_{3}+\mu_{7}, \\
\beta_{6}:= & \frac{1}{2}\left(\mu_{1}-\mu_{5}+\mu_{4}-\mu_{8}-\mu_{9}+\mu_{10}+\mu_{12}-\mu_{13}\right),
\end{aligned}
$$

the result eventually reads

$$
\begin{aligned}
{ }^{\text {odd }} \chi^{\alpha \beta}{ }_{\mu}^{\gamma \delta}{ }_{\nu}(g)= & \frac{\alpha}{\varkappa}\left[\beta_{4} \varepsilon^{\alpha \beta \gamma \delta} g_{\mu \nu}+\beta_{5} \varepsilon^{\alpha \beta[\gamma}{ }_{[\mu} \delta_{\nu]}^{\delta]}\right. \\
& \left.+\beta_{6}\left(\delta_{(\mu}^{[\alpha} \varepsilon^{\beta] \gamma \delta}{ }_{\nu)}-\delta_{(\mu}^{[\gamma} \varepsilon^{\delta] \alpha \beta}{ }_{\nu)}\right)\right] .
\end{aligned}
$$

Here $\alpha$ is dimensionless pseudoscalar. It is necessary in order to take into account the transformation law of this part under improper reflections.

Therefore, the most general parity-odd part of the constitutive tensor in the end boils down to just the three independent terms with $\beta_{4}, \beta_{5}$, and $\beta_{6}$. The $\beta_{4}$-term, being totally antisymmetric in $\alpha \beta \gamma \delta$, represents an $\beta_{4}$-axion, whereas the $\beta_{5}$-term describes a $\beta_{5}$-axion. They both represent reversible processes. In contrast, the $\beta_{6}$-term corresponds to irreversibility. When contracted by $F_{\alpha \beta}{ }^{\mu}$ and $F_{\gamma} \delta^{\nu}$, this term drops out from the general teleparallel Lagrangian. Moreover, one can reduce the number of independent terms in the Lagrangian by making use of the Nieh-Yan topological invariant $[41,42,46]$.

\subsection{The general case and its irreducible decomposition}

The general constitutive tensor

$$
\chi^{\alpha \beta}{ }_{\mu}{ }^{\gamma \delta}{ }_{\nu}(g)={ }^{\text {even }} \chi^{\alpha \beta}{ }_{\mu}{ }^{\gamma \delta}{ }_{\nu}(g)+{ }^{\text {odd }} \chi^{\alpha \beta}{ }_{\mu}{ }^{\gamma \delta}{ }_{\nu}(g)
$$

encompasses the parity-even piece of Eq. (139) and the parity-odd piece of Eq. (155),

Computing the irreducible parts, we find

$$
{ }^{[2]} \chi^{\alpha \beta}{ }_{\mu}{ }^{\gamma \delta}{ }_{\nu}(g)={ }^{[6]} \chi^{\alpha \beta}{ }_{\mu}{ }^{\gamma \delta}{ }_{v}(g)=0,
$$

whereas the nontrivial pieces read

$$
\begin{aligned}
& { }^{[1]} \chi^{\alpha \beta}{ }_{\mu}{ }^{\gamma \delta}{ }_{\nu}(g)= \\
& \frac{\sqrt{-g}}{\varkappa}\left[\beta_{1} g^{\gamma[\alpha} g^{\beta] \delta} g_{\mu \nu}+\left(\beta_{2}+\beta_{3}\right) \delta_{(\mu}^{[\alpha} g^{\beta][\gamma} \delta_{\nu)}^{\delta]}\right], \\
& { }^{[3]} \chi^{\alpha \beta}{ }_{\mu}{ }^{\gamma \delta}{ }_{\nu}(g)=\frac{\beta_{6}}{\varkappa}\left(\delta_{(\mu}^{[\alpha} \varepsilon^{\beta] \gamma \delta}{ }_{\nu)}-\delta_{(\mu}^{[\gamma} \varepsilon^{\delta] \alpha \beta}{ }_{\nu)}\right), \\
& { }^{[4]} \chi^{\alpha \beta}{ }_{\mu}{ }^{\gamma \delta}{ }_{\nu}(g)= \\
& \frac{1}{x}\left[\left(\beta_{2}-\beta_{3}\right) \sqrt{-g} \delta_{[\mu}^{[\alpha} g^{\beta][\gamma} \delta_{\nu]}^{\delta]}+\beta_{5} \varepsilon^{\alpha \beta[\gamma}{ }_{[\mu} \delta_{v]}^{\delta]}\right], \\
& { }^{[5]} \chi^{\alpha \beta}{ }_{\mu}{ }^{\gamma \delta}{ }_{\nu}(g)=\frac{\beta_{4}}{\varkappa} \varepsilon^{\alpha \beta \gamma \delta} g_{\mu \nu} .
\end{aligned}
$$

Summarizing, a general metric-dependent constitutive tensor encompasses two principal ${ }^{[1]} \chi$ and ${ }^{[4]} \chi$ parts, one axion ${ }^{[5]} \chi$ part, and one skewon ${ }^{[3]} \chi$ part.

Calculating the traces (105)-(108) of the constitutive tensor (156), we obtain

$m^{\alpha \beta}=\frac{3 \sqrt{-g}}{4 \varkappa}\left(2 \beta_{1}-3 \beta_{2}-\beta_{3}\right) g^{\alpha \beta}$,

and

$n^{\alpha \beta}=\frac{3 \sqrt{-g}}{4 \varkappa}\left(2 \beta_{1}-\beta_{2}-3 \beta_{3}\right) g^{\alpha \beta}$.

The antisymmetric tensors vanish, $k^{\alpha \beta}=l^{\alpha \beta}=0$. The parity odd terms drops out altogether in the trace building.

\subsection{Specific Lagrangians.}

\subsubsection{GR $R_{\|}$: the teleparallel equivalent of $G R$}

We found in the framework of the teleparallel equivalent $\mathrm{GR}_{||}$of general relativity (GR) for the $\beta$ 's in [31, Eq. (88)] $]^{6}$,

$$
\left.\begin{array}{lll}
\beta_{1}=-1, & \beta_{2}=-4, & \beta_{3}=2 \\
\beta_{4}=0, & \beta_{5}=0, & \beta_{6}=0
\end{array}\right\} \quad \mathrm{GR}_{\|} .
$$

Accordingly, the three $\beta$ 's are related to GR in a quite definite way, and we get a feeling for their interpretation.

The $\mathrm{GR}_{||}$Lagrangian is distinguished from the other teleparallelism Lagrangians as being locally Lorentz invariant, see, e.g., Cho [11] and Müller-Hoissen and Nitsch [40]. Any other additional term of even parity in the gravitational Lagrangian removes this local invariance.

Ferrarro and Guzmán [14] called the constitutive tensor $\chi^{\alpha \beta}{ }_{\mu}^{\gamma \delta}{ }_{v}(g)$ of (156) together with the parameters of (164)

\footnotetext{
6 There occurred an computing error. The correct values in the old conventions are $\beta_{1}^{\text {old }}=-\frac{1}{4}, \beta_{2}^{\text {old }}=\frac{1}{2}, \beta_{3}^{\text {old }}=-\frac{1}{4}$.
} 
the supermetric. In fact, up to a conventional factor of -2 , our result agrees with their Eq. (17) in [14].

\subsubsection{Von der Heyde Lagrangian}

Earlier, different parity even pieces of torsion square Lagrangians were compared by Muench et al. [39], see also the literature cited therein. A particular role played in these considerations the torsion square piece of a Lagrangian of von der Heyde [24]. Its constitutive tensor [17] reads

${ }^{\mathrm{vdH}} \chi^{\alpha \beta}{ }_{\mu}{ }^{\gamma \delta}{ }_{\nu}=-\frac{2 \sqrt{-g}}{\varkappa}\left(g^{\gamma[\alpha} g^{\beta] \delta} g_{\mu \nu}+2 \delta_{\mu}^{[\alpha} g^{\beta][\gamma} \delta_{\nu}^{\delta]}\right)$.

For the corresponding constitutive law, we have

$$
\begin{aligned}
\check{H}^{\alpha \beta}{ }_{\mu} & =\frac{1}{2}{ }^{\mathrm{vdH}} \chi^{\alpha \beta}{ }_{\mu}{ }^{\gamma \delta}{ }_{\nu} F_{\gamma \delta}{ }^{\nu} \\
& =-\frac{\sqrt{-g}}{\varkappa}\left(F_{\mu}^{\alpha \beta}{ }_{\mu}+2 \delta_{\mu}^{[\alpha} F^{\beta]}{ }^{\gamma}\right) .
\end{aligned}
$$

This law, in the teleparallel case, leads already to the correct Newtonian approximation. The constitutive tensor (165) carries the following $\beta$-values:

$$
\left.\begin{array}{lll}
\beta_{1}=-2, & \beta_{2}=-4, & \beta_{3}=0 \\
\beta_{4}=0, & \beta_{5}=0, & \beta_{6}=0
\end{array}\right\} \quad \mathrm{vdH}
$$

As we saw, the teleparallel equivalent $\mathrm{GR}_{||}$has a slightly different constitutive law (164).

\subsubsection{Torsion-square Lagrangian in nonlocal gravity $(N L G)$}

In a nonlocal extension of GR - Nonlocal Gravity (NLG) - the starting point $[19,20]$ was the parameter set of $\mathrm{GR}_{\|}$, namely (164). Later, Mashhoon found it necessary, to enlarge it by adding a supplementary piece proportional to the axial ${ }^{7}$ torsion ${ }^{(3)} F_{\alpha \beta}{ }^{\mu}$, see [37, Eq. (6.109)]. This yields, with an unknown dimensionless parameter $\breve{p}$,

$$
\left.\begin{array}{lll}
\beta_{1}=-1, & \beta_{2}=-4, & \beta_{3}=2, \\
\beta_{4}=-\frac{\check{p}}{6}, & \beta_{5}=-4 \frac{\check{p}}{6}, & \beta_{6}=2 \frac{\check{p}}{6} .
\end{array}\right\} \text { Mashh. }
$$

It is amazing that $\left(\beta_{4}, \beta_{5}, \beta_{6}\right)=\frac{\check{p}}{6}\left(\beta_{1}, \beta_{2}, \beta_{3}\right)$. Probably this means that Mashhoon's additional parity odd term is locally Lorentz invariant. Incidentally, there is a conventional factor between Mashhoon's $\check{p}$ and ours.

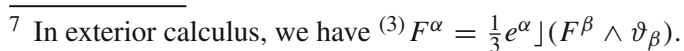

According to Mashhoon, ${ }^{8}$ “....the mere fact of postulating a nonlocal constitutive relation violates local Lorentz invariance." Still, the version of NLG of 2009 is based on the reversible and locally Lorentz invariant parameter set (164) and Mashhoon's version corresponds to an irreversible and possibly also locally Lorentz invariant parameter set, see the 2nd line of (168).

\section{Propagation of gravitational waves}

In this section, we discuss the wave propagation in our premetric teleparallel theory (TG) in linear approximation.

\subsection{Geometric optics approximation}

We start with the source-free field equations

$d H_{\alpha}=0 \quad$ and $\quad d F^{\alpha}=0$.

Notice that the source term $\Sigma$ in (2) contains two independent expressions: the matter energy-momentum current ${ }^{(\mathrm{m})} \Sigma$ and the gravitational energy-momentum current ${ }^{(\vartheta)} \Sigma$. In matterfree regions, we have ${ }^{(\mathrm{m})} \Sigma=0$. Similar to standard GR, we will assume, in addition, ${ }^{(\vartheta)} \Sigma=0$. This requirement is applicable for small waves and means linearization of the field equations.

In the geometric optics approximation (and, equivalently, in Hadamard's approach, see [22]), one derives the linear system

$\check{H}^{\mu \nu}{ }_{\alpha} q_{\nu}=0 \quad$ and $\quad \epsilon^{\mu \nu \rho \sigma} F_{\rho \sigma}^{\alpha} q_{\nu}=0$.

Here the components $q_{v}$ of the wave covector are determined by the differential of the phase $\varphi$ function of the wave: $d \varphi=$ $q_{v} \vartheta^{\nu}$.

The second equation of (170) has the solution

$F_{\rho \sigma}{ }^{\alpha}=A_{\rho}{ }^{\alpha} q_{\sigma}-A_{\sigma}{ }^{\alpha} q_{\rho}$,

with an arbitrary tensor $A_{\beta}{ }^{\alpha}$. It is a gravitational analog of the electromagnetic potential. We observe the gauge invariance of this gravitational potential. An expression of the form $A_{\beta}{ }^{\alpha}=q_{\beta} C^{\alpha}$ does not contribute to the field strength $F_{\rho \sigma}{ }^{\alpha}$. In other words, the model is invariant under the transformations

$A_{\beta}{ }^{\alpha} \rightarrow A_{\beta}{ }^{\alpha}+q_{\beta} C^{\alpha}$,

with an arbitrary vector $C^{\alpha}$.

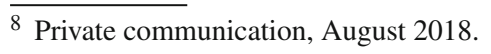


We substitute (171) into (170) and use the constitutive relation

$\check{H}_{\alpha}^{\mu \nu}=\frac{1}{2} \chi^{\mu \nu}{ }_{\alpha}^{\rho \sigma}{ }_{\beta} F_{\rho \sigma}{ }^{\beta}$.

This yields the characteristic equation

$M_{\alpha}^{\mu}{ }_{\alpha}^{\nu} A_{\nu}^{\beta}=0$

with fourth order characteristic tensor

$M_{\alpha \beta}^{\mu}{ }_{\alpha}^{\nu}:=\chi_{\alpha}^{\mu \rho}{ }^{\nu \sigma}{ }_{\beta} q_{\rho} q_{\sigma}$.

Inserting here the decomposition (38) of the constitutive tensor, we observe that the axion-type parts ${ }^{[5]} \chi^{\mu \rho}{ }_{\alpha}{ }^{\nu \sigma_{\beta}}$ and ${ }^{[6]} \chi^{\mu \rho}{ }_{\alpha}{ }^{v \sigma}{ }_{\beta}$ do not enter the tensor $M^{\mu}{ }_{\alpha}{ }^{\nu}{ }_{\beta}$. Consequently, these parts do not contribute to the wave propagation as it was already outlined in Table 1.

In the reversible case, the characteristic tensor $M^{\mu}{ }_{\alpha}{ }^{\nu} \beta$ satisfies the symmetry relations

$M_{\alpha}^{\mu}{ }^{v}{ }_{\beta}=M^{v}{ }_{\beta}^{\mu}{ }_{\alpha}$.

A generalized Fresnel equation can be derived as the condition for the solvability of the Eq. (174) along the lines of the algebraic computations of Itin [30].

\subsection{Dispersion relation: general facts}

Observe that the characteristic equation

$E_{\alpha}^{\mu}=0$, with $E_{\alpha}^{\mu}:=M^{\mu}{ }_{\alpha}{ }^{\nu}{ }_{\beta} A_{\nu}{ }^{\beta}$,

represents 16 equations for the 16 variables $A_{v}{ }^{\beta}$.

For a compact representation of this system, we denote a pair of upper and lower indices by a multi-index

$\left\{\begin{array}{c}\mu \\ \alpha\end{array}\right\}=I \quad I=1, \ldots 16$.

In this notation, the system (177) reads

$E^{I}=M^{I J} A_{J}=0$.

The gauge transformation (172) can be rewritten as

$A_{J} \rightarrow A_{J}+Q_{J}, \quad$ where $\quad Q_{J}=q_{\beta} C^{\alpha}$.

The 4 linearly independent vectors $C^{\alpha}$ imply that there are likewise 4 linearly independent vectors $Q_{J}$. The identities

$M_{\alpha}^{\mu}{ }_{\alpha}^{\nu} q_{\mu}=M_{\alpha}^{v}{ }^{\mu}{ }_{\beta} q_{\mu}=0$ translate into

$M^{I J} Q_{J}=M^{J I} Q_{J}=0$.

These equations can be understood as 4 linear relations between the rows (and the columns) of the matrix $M^{I J}$. Consequently, the matrix $M^{I J}$ has rank $12=16-4$. The condition for the existence of non-trivial solutions of Eq. (179) reads now as

${ }^{(4)} \operatorname{Adj}(M)=0$.

We used here the fourth order adjoint of the matrix $M^{I J}$. It can be constructed by evaluating the determinants of the matrices that are left in the $16 \times 16$ matrix $M^{I J}$ by removing four rows and four columns. Consequently ${ }^{(4)} \operatorname{Adj}(M)$ is a set of 12th order polynomials of the entries $M^{I J}$. Accordingly, it is a set of 12th order polynomials of the variables $q_{\alpha}$. The 4th order adjoint can be written as a tensor with eight free indices:

${ }^{(4)} \operatorname{Adj}(M)_{I_{1} \cdots I_{4} J_{1} \cdots J_{4}}=0$.

In electromagnetism with only one gauge invariance constraint, there appears the 1 st order adjoint matrix $\operatorname{Adj}(M)_{\alpha \beta}$. This matrix is expressed as a scalar function multiplied by a tensor product of $q_{\alpha}$, namely $\operatorname{Adj}(M)_{\alpha \beta}=\lambda(q) q_{\alpha} q_{\beta}$. Thus, the electromagnetic dispersion relation takes the form $\lambda(q)=0$.

Similarly, in the case of the gravitational equation (184), we have

${ }^{(4)} \operatorname{Adj}(M)_{I_{1} \cdots I_{4} J_{1} \cdots J_{4}}=\Lambda(Q) Q_{I_{1}} \cdots Q_{I_{4}} Q_{J_{1}} \cdots Q_{J_{4}}=0$.

Thus, the gravitational dispersion relation takes the form

$\Lambda(q)=0$.

Here $\Lambda(q)$ is a homogeneous polynomial of the order $16=$ $24-8$ in the variable $q_{\alpha}$. We recall that in the electromagnetic case, the corresponding form is of 4 th order. This fact yields birefringence in the wave propagation. In 3-dimensional elasticity theory, the dispersion relation is of 6th order, with 3 different waves in general. In our generalized gravitational model, there are 8 different waves in general.

In the simplest case, Eq. (186) reads $\left(g^{\alpha \beta} q_{\alpha} q_{\beta}\right)^{8}=0$. We discuss the metrical case in Sect. 6.

\subsection{Dispersion relation decomposed}

In order to clarify the nature of the Eq. (177), we decompose the solution under the $\operatorname{GL}(4, \mathbb{R})$ irreducibly into the scalar 
$A \delta_{\alpha}^{\beta}$ and the traceless part $A_{\alpha}{ }^{\beta}$,

$A_{\alpha}{ }^{\beta}=A_{\alpha}{ }^{\beta}+A \delta_{\alpha}^{\beta}$, with $A:=\frac{1}{4} A_{\gamma}^{\gamma}$ and $A_{\alpha}^{\alpha}=0$.

Consequently, Eq. (177) decomposes into

$E_{\alpha}^{\mu}=M_{\alpha}^{\mu}{ }^{v} \beta A_{\nu}{ }^{\beta}+M_{\alpha}^{\mu}{ }^{\beta}{ }_{\beta} A=0$.

A similar decomposition can be performed with $E^{\mu}$. Thus, we find the $1+15$ equations

$E_{\alpha}^{\alpha}=0 \quad$ and $\quad E^{\mu}{ }_{\alpha}-\frac{1}{4} E_{\gamma}^{\gamma} \delta_{\alpha}^{\mu}=0$

or, explicitly, one equation

$M^{\alpha}{ }_{\alpha}{ }^{v} \beta A_{v}{ }^{\beta}+M_{\alpha}^{\alpha}{ }_{\alpha}^{\beta} A=0$

and 15 equations

$$
\begin{aligned}
& \left(M_{\alpha}^{\mu}{ }_{\alpha}^{\nu}-\frac{1}{4} \delta_{\alpha}^{\mu} M_{\gamma}^{\gamma}{ }^{\nu} \beta\right) A_{v}^{\beta} \\
& +\left(M_{\alpha}^{\mu}{ }^{\beta} \beta-\frac{1}{4} \delta_{\alpha}^{\mu} M^{\gamma}{ }_{\gamma}^{\beta} \beta\right) A=0 .
\end{aligned}
$$

Substituting (190) into (191), we derive the algebraic system for the traceless variable:

$N^{\mu}{ }_{\alpha}{ }^{\nu} A_{v}{ }^{\beta}=0$

where we introduced

$N^{\mu}{ }_{\alpha}{ }^{\nu} \beta:=M^{\mu}{ }_{\alpha}{ }^{\nu}{ }_{\beta} M^{\rho}{ }_{\rho}{ }^{\sigma}{ }_{\sigma}-M^{\mu}{ }_{\alpha}{ }^{\rho}{ }_{\rho} M^{\sigma}{ }_{\sigma}{ }^{v}{ }_{\beta}$.

The latter tensor is apparently traceless for both pairs of indices:

$N_{\alpha \rho}^{\mu}{ }_{\alpha}^{\rho}=0, \quad N^{\sigma}{ }_{\sigma}^{\nu}{ }_{\beta}=0$.

Accordingly, we find a system of 15 algebraic equations for 15 variables $A_{\nu}{ }^{\mu}$. After one solves the homogeneous equation (192), one immediately finds the scalar $A$ from (190).

\subsection{Scalar waves as a special case}

We consider now a special case of pure scalar waves. Let the field $A_{v}{ }^{\beta}$ be identically zero: $A_{v}{ }^{\beta}=0$. Hence, we are left with a system of two scalar equations

$$
\left(M_{\alpha}^{\mu}{ }_{\alpha}{ }_{\beta}-\frac{1}{4} \delta_{\alpha}^{\mu} M_{\gamma}^{\gamma}{ }_{\beta}{ }^{\prime}\right) A=0, \quad M_{\alpha}^{\alpha}{ }_{\beta}{ }_{\beta} A=0 .
$$

The second equation has a non-trivial solution only if $M^{\alpha}{ }_{\alpha}{ }_{\beta}$ $=0$. Consequently we have a dispersion relation

$\chi_{\alpha}^{\alpha \rho}{ }_{\beta \sigma}^{\beta \sigma} q_{\rho} q_{\sigma}=0$.

Using the double-trace tensor (105), we can write it as

$m^{\alpha \beta} q_{\alpha} q_{\beta}=0$

In the metric-dependent case (162), the tensor $m^{\alpha \beta}$, up to a factor, coincides with the Lorentz metric tensor $g^{\alpha \beta}$. Thus we recover the standard light cone of general relativity,

$g^{\alpha \beta} q_{\alpha} q_{\beta}=0$

\subsection{A separable case}

We assume now that two terms in the left-hand side of Eqs. (190) and (191) vanish independently. In other words, we assume that the system separates into two independent subsystems. In [28], a similar type of consideration allowed to extract the teleparallel equivalent of GR from a set of metric based models. Thus we have 15 equations for 15 variables

$\left(M_{\alpha}^{\mu}{ }_{\alpha}^{\nu} \beta-\frac{1}{4} \delta_{\alpha}^{\mu} M_{\gamma}^{\gamma}{ }^{\nu} \beta\right) A_{\nu}{ }^{\beta}=0$.

and 1 equation for 1 variable

$M_{\alpha}^{\alpha}{ }_{\beta} A=0$

\subsection{Gauge conditions}

In electromagnetism, gauge invariance can be restricted by applying a gauge fixing condition. The Lorenz gauge condition is the unique diffeomorphism invariant expression. For wave solutions, it takes the form $A_{\alpha} q^{\alpha}=0$. Note that this condition can be formulated only on a metric manifold.

In teleparallel theory, we are able to formulate a similar condition in a premetric form:

$A_{v}^{\beta} q_{\beta}=0$.

A more general gauge condition can be proposed in the form of

$K_{1} A_{\alpha}^{\beta} q_{\beta}+K_{2} A_{\beta}^{\beta} q_{\alpha}=0$.

Here $K_{1}$ and $K_{2}$ are arbitrary constants. The exceptional case for $K_{1}=1$ and $K_{2}=-1$, namely

$A_{\alpha}^{\beta} q_{\beta}-A_{\beta}^{\beta} q_{\alpha}=0$

is invariant under the gauge transformation $A_{\alpha}{ }^{\beta} \rightarrow A_{\alpha}{ }^{\beta}+$ $q_{\alpha} C^{\beta}$ and, accordingly, does not represent a gauge condition. 


\section{Gravitational waves in a metric model}

Let us now specialize to the metric-dependent constitutive tensor (156). The analysis of the wave propagation in the geometric optics approximation is straightforward. Here we will confine our attention to the parity-even case (139). Recently a related paper was published by Hohmann et al. [27].

\subsection{Characteristic system}

We substitute the constitutive tensor (139) with its parity even pieces into (175). As a result, $M^{\beta}{ }_{\alpha}{ }^{\nu}{ }_{\mu}(g)=\chi^{\beta \gamma}{ }_{\alpha}{ }^{\nu \rho}{ }_{\mu}(g) \times$ $q_{\gamma} q_{\rho}$ takes the form

$$
\begin{aligned}
& M_{\alpha}^{\beta}{ }_{\alpha}{ }_{\mu}(g)=\frac{\sqrt{-g}}{4 \varkappa}\left\{2 \beta_{1} g_{\alpha \mu}\left(g^{\beta v} q^{2}-q^{v} q^{\beta}\right)\right. \\
& -\beta_{2}\left[\left(q^{2} \delta_{\alpha}^{\beta}-q^{\beta} q_{\alpha}\right) \delta_{\mu}^{v}-\left(q^{v} \delta_{\alpha}^{\beta}-g^{\beta v} q_{\alpha}\right) q_{\mu}\right] \\
& \left.-\beta_{3}\left[\left(q^{2} \delta_{\mu}^{\beta}-q^{\beta} q_{\mu}\right) \delta_{\alpha}^{v}-\left(q^{v} \delta_{\mu}^{\beta}-g^{\beta v} q_{\mu}\right) q_{\alpha}\right]\right\} .
\end{aligned}
$$

We can immediately derive the contractions

$$
M^{\beta}{ }_{\alpha}{ }^{\nu}{ }_{\nu}=M^{\nu}{ }_{\nu}{ }^{\beta}{ }_{\alpha}=\frac{\sqrt{-g}}{4 \varkappa}\left(2 \beta_{1}-3 \beta_{2}-\beta_{3}\right)\left(q^{2} \delta_{\alpha}^{\beta}-q^{\beta} q_{\alpha}\right),
$$

where $q^{2}:=g^{\alpha \beta} q_{\alpha} q_{\beta}$. Consequently,

$M_{\alpha}^{\alpha}{ }^{\nu}{ }_{\nu}=\frac{3 \sqrt{-g}}{4 \varkappa}\left(2 \beta_{1}-3 \beta_{2}-\beta_{3}\right) q^{2}$.

Furthermore, we verify that the tensor (204) has the following properties:

$$
\begin{aligned}
M_{\alpha}^{\beta}{ }^{\nu}{ }_{\mu} q_{\nu} & =0, \quad M^{\beta}{ }_{\alpha}{ }^{\nu}{ }_{\mu} q_{\beta}=0, \\
M^{\beta}{ }_{\alpha}{ }^{\nu}{ }_{\mu} q^{\mu} & =\frac{\sqrt{-g}}{4 \varkappa}\left(2 \beta_{1}-\beta_{2}-\beta_{3}\right)\left(q^{2} g^{\beta \nu}-q^{\beta} q^{\nu}\right) q_{\alpha}, \\
M^{\beta}{ }_{\alpha}{ }^{\nu}{ }_{\mu} q^{\alpha} & =\frac{\sqrt{-g}}{4 \varkappa}\left(2 \beta_{1}-\beta_{2}-\beta_{3}\right)\left(q^{2} g^{\beta \nu}-q^{\beta} q^{\nu}\right) q_{\mu} .
\end{aligned}
$$

Interestingly, for the $\mathrm{GR}_{||}$case of (164), the right-hand sides of (208) and (209) vanish, i.e., the tensor $M^{\beta}{ }_{\alpha}{ }^{\nu}{ }_{\mu}$ turns out to be transversal to the wave covector $q_{\mu}$ in all four indices.

Taking into account these properties, the wave propagation system (177) yields

$$
\left(2 \beta_{1}-\beta_{2}-\beta_{3}\right)\left(q^{2} g^{\beta \nu}-q^{\beta} q^{\nu}\right) q_{\mu} A_{\nu}{ }^{\mu}=0 .
$$

In the generic case, when $2 \beta_{1}-\beta_{2}-\beta_{3} \neq 0$, we find the relation

$q^{2} A_{\beta}{ }^{\mu} q_{\mu}=q_{\beta} A_{\nu}{ }^{\mu} q_{\mu} q^{\nu}$,

which implies

$q^{2} \not_{\beta}{ }^{\mu} q_{\mu}=q_{\beta} \not_{\nu}{ }^{\mu} q_{\mu} q^{\nu}$.

We will use this in subsequent derivations.

Let us now turn to the decomposed equations (191) and (192), Using (205), (206), and $\not_{v}{ }^{\nu}=0$, we can recast (191) into

$3 q^{2} A=A_{\nu}{ }^{\mu} q_{\mu} q^{\nu}$.

This is derived for the generic case by assuming $2 \beta_{1}-3 \beta_{2}-$ $\beta_{3} \neq 0$, otherwise (191) is trivial.

Finally, we turn to the traceless part (192) of the wave propagation equation. Substituting (204)-(206) into (193), and then making use of (212), we can rewrite (192) in the equivalent form

$$
\begin{aligned}
6 & \beta_{1} q^{2}\left(q^{2} A_{\alpha}^{\beta}-q^{\beta} q_{\gamma} A^{\gamma}{ }_{\alpha}\right) \\
& -3 \beta_{3} q^{2}\left(q^{2} \not_{\alpha}{ }^{\beta}-q_{\alpha} q^{\gamma} A_{\gamma}{ }^{\beta}\right) \\
& +\left(2 \beta_{1}-\beta_{3}\right)\left(q^{2} \delta_{\alpha}^{\beta}-q^{\beta} q_{\alpha}\right) \not_{\nu}{ }^{\mu} q_{\mu} q^{\nu}=0 .
\end{aligned}
$$

Quite remarkably, the coupling constant $\beta_{2}$ does not contribute.

Decomposing (214) into symmetric and antisymmetric parts, we obtain the two equations

$$
\begin{aligned}
& \left(2 \beta_{1}-\beta_{3}\right)\left\{3\left(q^{2} \delta_{\alpha}^{\mu}-q^{\mu} q_{\alpha}\right)\left(q^{2} \delta_{\beta}^{\nu}-q^{\nu} q_{\beta}\right)\right. \\
& \left.\quad-\left(q^{2} g_{\alpha \beta}-q_{\alpha} q_{\beta}\right) q^{\mu} q^{\nu}\right\} A_{(\mu \nu)}=0, \\
& \left(2 \beta_{1}+\beta_{3}\right)\left(q^{2} \delta_{\alpha}^{\mu}-q^{\mu} q_{\alpha}\right)\left(q^{2} \delta_{\beta}^{\nu}-q^{\nu} q_{\beta}\right) A_{[\mu \nu]}=0 .
\end{aligned}
$$

Two important observations are in order. Firstly, we see that the symmetric $A_{(\mu \nu)}$ and skew-symmetric $A_{[\mu \nu]}$ variables decouple from each other and are governed by two separate dynamical equations. Secondly, the symmetric $A_{(\mu v)}$ mode remains the only one when coupling constants satisfy $2 \beta_{1}=$ $-\beta_{3}$ and, similarly, the antisymmetric $A_{[\mu \nu]}$ mode remains the only one when the coupling constants satisfy $2 \beta_{1}=\beta_{3}$.

In the generic case (when $2 \beta_{1} \neq-\beta_{3}$ and $2 \beta_{1} \neq \beta_{3}$ ), both modes are dynamical. In principle, one can proceed by deriving the Fresnel equations for each mode in a way similar to classical electrodynamics. However, one can choose an alternative way and analyze the propagation equations by 
using the method of spin-projection operators. The corresponding tools were earlier developed in metric gravity [52] and in its teleparallel formulation [40]. Their application is straightforward. Ultimately we conclude that the wave equations describe the massless spin- 0 , spin- 2 and spin- 1 modes propagating along the light cones $q^{2}=0$.

\subsection{Waves in $\mathrm{GR}_{\|}$, the teleparallel equivalent of GR}

In order to understand more clearly what happens in $\mathrm{GR}_{\|}$, it is instructive to analyze the wave propagation equation from scratch. Plugging (204) into (175), we find for (174), after some straightforward algebra and lowering the index $\mu$,

$$
\begin{aligned}
\left(2 \beta_{1}-\beta_{2}-\beta_{3}\right) \\
\times\left\{4 A\left(q^{2} g_{\alpha \beta}-q_{\alpha} q_{\beta}\right)-g_{\alpha \beta} q^{\mu} q^{\nu} A_{\mu \nu}+q_{\alpha} A_{\beta \gamma} q^{\gamma}\right\} \\
-\left(2 \beta_{1}+\beta_{3}\right)\left\{q^{2} A_{[\alpha \beta]}-q_{\alpha} q^{\gamma} A_{[\gamma \beta]}-q_{\beta} q^{\gamma} A_{[\alpha \gamma]}\right\} \\
+\left(2 \beta_{1}-\beta_{3}\right)\left\{q^{2} A_{(\alpha \beta)}-q_{\alpha} q^{\gamma} A_{(\gamma \beta)}-q_{\beta} q^{\gamma} A_{(\alpha \gamma)}\right. \\
\left.-4 A\left(q^{2} g_{\alpha \beta}-q_{\alpha} q_{\beta}\right)+g_{\alpha \beta} q^{\mu} q^{\nu} A_{\mu \nu}\right\}=0 .
\end{aligned}
$$

Contracting with $g^{\alpha \beta}$, we find

$$
\left(2 \beta_{1}-3 \beta_{2}-\beta_{3}\right)\left(4 A q^{2}-q^{\mu} q^{\nu} A_{\mu \nu}\right)=0,
$$

whereas contraction with $q^{\alpha}$ yields

$$
\left(2 \beta_{1}-\beta_{2}-\beta_{3}\right)\left(-q_{\beta} q^{\mu} q^{\nu} A_{\mu \nu}+q^{2} A_{\beta \gamma} q^{\gamma}\right)=0 .
$$

In the generic case, we recover the earlier findings (211) and (210). Making use of this, we see that the first line in (217) vanishes, whereas the remaining equation reduces to the system (215) and (216).

However, $\mathrm{GR}_{||}$represents a very special case when the coupling constants (164) are such that both $2 \beta_{1}-\beta_{2}-\beta_{3}=0$ and $2 \beta_{1}+\beta_{3}=0$. As a result, the first two lines of (217) disappear, and the propagation equation has only a symmetric traceless part. The bottom line is that in $\mathrm{GR}_{\|}$Eq. (217) reduces to

$q^{2} h_{\alpha \beta}-q_{\alpha} q^{\gamma} h_{\gamma \beta}-q_{\beta} q^{\gamma} h_{\alpha \gamma}=0$,

after we introduce the familiar variable

$h_{\alpha \beta}:=A_{(\alpha \beta)}-2 A g_{\alpha \beta}=A_{(\alpha \beta)}-\frac{1}{2} g_{\alpha \beta} A_{\gamma}^{\gamma}$.

Accordingly, we indeed recover the result of GR with the massless spin-2 graviton propagating along the light cone. In other words, $\mathrm{GR}_{||}$is completely consistent with Einstein's theory with respect to the propagation of gravitational waves.

\section{Conclusions and outlook}

Recently, in Ref. [29], we developed a novel framework for a premetric teleparallel theory of gravity (TG). Here we continue this study on TG by paying attention specifically to TG models with a general local and linear constitutive law.

In Sect. 2, we constructed the Tonti diagram of TG which explicitly displays the generic structure of the theory.

Our main new results are presented in Sect. 3 where the irreducible decomposition of the premetric constitutive tensor is established in full detail. This issue was not analysed in the earlier literature. Here we considered two types of such decompositions with respect to the permutation group, namely one related to $S_{4} \times S_{2}$ and the other one with respect to $S_{6}$. The relations between these two decompositions are explicitly derived and the physical meaning of the different irreducible pieces clarified.

After establishing for the constitutive tensor these premetric results, we turned in Sect. 4 to a special case: The spacetime continuum is supposed to carry a metric tensor. We constructed the most general metric dependent constitutive tensor that is cubic in the metric tensor. It includes both, parity even and parity odd parts. Thereby extending earlier results, we for the first time obtained the most general family of teleparallel consitutive tensors. In particular, a parity odd Lagrangian was newly found, and its physical interpretation fixed.

In Sect. 5, the propagation of gravitational waves are derived for the premetric case in the geometrical optics approximation. Additional propagating modes of spin 1 and 0 are extracted. Our results are generally consistent with the recent findings reported in [27].

Subsequently, in Sect. 6, the metric case is addressed. The GR limit is naturally embedded in our formalism.

The results presented here can serve as a basis for the study of expanded gravitational models including axion and skewon effects. In particular, violation of Lorentz invariance can be meaningfully addressed in our premetric set-ups.

Acknowledgements We are grateful to Enzo Tonti (Trieste) for help in creating our Tonti diagram. Moreover, we thank Bahram Mashhoon (Tehran) for most helpful remarks in the context of nonlocal gravity (NLG). Remarks of María José Guzmán (Buenos Aires), Manuel Hohmann (Tartu), José Maluf (Brasilia), and James Nester (ChungLi) are gratefully acknowledged. For Y.N.O. this work was partially supported by the Russian Foundation for Basic Research (Grant no. 1602-00844-A). J.B. is grateful for a Vanier Canada Graduate Scholarship administered by the Natural Sciences and Engineering Research Council of Canada as well as for the Golden Bell Jar Graduate Scholarship in Physics by the University of Alberta.

Open Access This article is distributed under the terms of the Creative Commons Attribution 4.0 International License (http://creativecomm ons.org/licenses/by/4.0/), which permits unrestricted use, distribution, and reproduction in any medium, provided you give appropriate credit to the original author(s) and the source, provide a link to the Creative 
Commons license, and indicate if changes were made.

Funded by SCOAP ${ }^{3}$.

\section{Appendix: Young decomposition}

Here we briefly explain how to determine the $\operatorname{GL}(4, \mathbb{R})$ decomposition of the order- $\left(\begin{array}{l}0 \\ 6\end{array}\right)$ constitutive tensor $\check{\chi}_{\alpha \beta \mu \gamma \delta \nu}$. The decomposition rules for tensors of other valence, say order- 2 or order- 4 , can easily be derived. In four dimensions, the decomposition of $\check{\chi}_{\alpha \beta \mu \gamma \delta \nu}$ with the symmetry properties

$\check{\chi}_{(\alpha \beta) \mu \gamma \delta v}=0, \quad \check{\chi}_{\alpha \beta \mu(\gamma \delta) v}=0$,

consists of the following seven pieces:

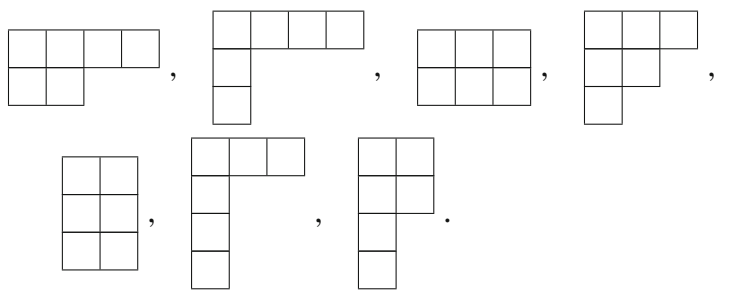

Let us label them from $\lambda_{1}$ to $\lambda_{7}$, respectively. Each diagram $\lambda_{i}(i=1 \ldots 7)$ has corresponding dimensions according to

$$
\begin{aligned}
\operatorname{dim} V^{\lambda} & =\prod_{(\alpha, \beta) \in \lambda} \frac{n+\beta-\alpha}{\operatorname{hook}(\alpha, \beta)}, \\
\operatorname{dim} S_{6}^{\lambda} & =f^{\lambda}=\frac{6 !}{\prod_{x \in \lambda} \operatorname{hook}(x)} .
\end{aligned}
$$

We find

$$
\begin{aligned}
& \operatorname{dim} V^{\lambda_{1}}=126, \quad \operatorname{dim} V^{\lambda_{2}}=70, \quad \operatorname{dim} V^{\lambda_{3}}=50, \\
& \operatorname{dim} V^{\lambda_{4}}=64, \quad \operatorname{dim} V^{\lambda_{5}}=10, \quad \operatorname{dim} V^{\lambda_{6}}=10, \\
& \operatorname{dim} V^{\lambda 7}=6,
\end{aligned}
$$

as well as

$$
\begin{array}{lll}
\operatorname{dim} S_{6}^{\lambda_{1}}=9, & \operatorname{dim} S_{6}^{\lambda_{2}}=10, & \operatorname{dim} S_{6}^{\lambda_{3}}=5, \\
\operatorname{dim} S_{6}^{\lambda_{4}}=16, & \operatorname{dim} S_{6}^{\lambda_{5}}=5, & \operatorname{dim} S_{6}^{\lambda_{6}}=10, \\
\operatorname{dim} S_{6}^{\lambda_{7}}=9 . &
\end{array}
$$

With the relevant Young diagrams now given, we can extract the projection operators in the standard fashion and arrive at the irreducible decomposition of $\chi_{\alpha \beta \gamma \mu \nu \rho}$.

For each $\lambda_{i}$ we may extract projection operators in the standard way: we can fill $f^{\lambda_{i}}$ copies of the $\lambda_{i}$ Young diagram with numbers to create all allowed Young tableaux, and then create the corresponding projection operator onto that subspace by summing over the induced Young symmetrizers:

$$
\begin{aligned}
\mathbb{P}_{p}^{\lambda_{i}} & :=\frac{f^{\lambda_{i}}}{p !} \sum_{j=1}^{f^{\lambda_{i}}} \mathbb{P}\left(Y_{j}^{\lambda_{i}}\right), \\
{ }^{(I)} \check{\chi}_{\alpha \beta \mu \gamma \delta \nu}: & =\mathbb{P}_{p}^{\lambda_{I}}\left(\check{\chi}_{\alpha \beta \mu \gamma \delta \nu}\right),
\end{aligned}
$$

where $\mathbb{P}\left(Y_{j}^{\lambda_{i}}\right)$ is the Young symmetrizer corresponding to the $j$-th Young tableaux of $\lambda_{i}$, and ${ }^{(I)} \check{\chi}_{\alpha \beta \mu \nu \delta \nu}$ denotes the $I$-th irreducible piece of the constitutive tensor. The Young tableaux with non-trivial symmetrizers are as follows:

$$
\begin{aligned}
& \lambda_{1}: \begin{array}{|l|l|l|l|}
\hline 1 & 3 & 5 & 6 \\
\hline 2 & 4 &
\end{array}, \begin{array}{|l|l|l|l|}
\hline & 3 & 4 & 6 \\
\hline 2 & 5 &
\end{array}
\end{aligned}
$$

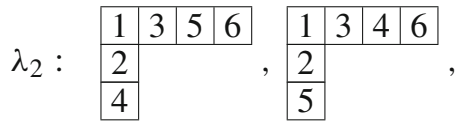

$$
\begin{aligned}
& \lambda_{3}: \begin{array}{|l|l|l|}
\hline 1 & 3 & 5 \\
\hline 2 & 4 & 6 \\
\hline
\end{array}, \begin{array}{|l|l|l|}
\hline 1 & 3 & 4 \\
\hline 2 & 5 & 6 \\
\hline
\end{array},
\end{aligned}
$$

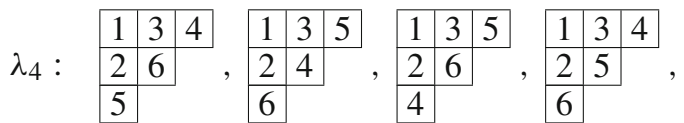

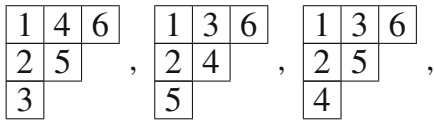

$$
\begin{aligned}
& \lambda_{5}: \begin{array}{|l|l|l|l|}
\hline 1 & 4 \\
\hline 2 & 5 \\
\hline 3 & 6 \\
\hline
\end{array}, \begin{array}{|l|l|l|}
\hline 1 & 3 \\
\hline 2 & 4 \\
\hline 5 & 6 \\
\hline
\end{array}, \begin{array}{|l|l|}
\hline 1 & 3 \\
\hline 4 & 5 \\
\hline 4 & 6 \\
\hline
\end{array}
\end{aligned}
$$

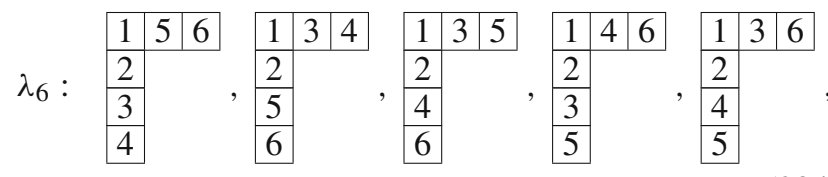

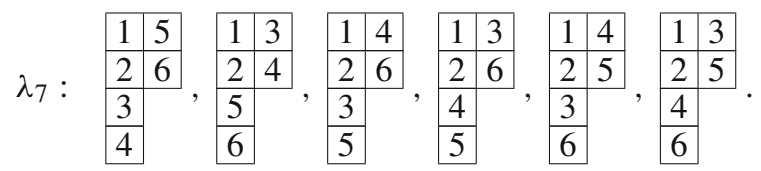

For example, $\mathbb{P}\left(Y_{6}^{\lambda_{4}}\right)$ can be computed as follows:

$$
\begin{aligned}
\mathbb{P}\left(Y_{6}^{\lambda_{4}}\right)= & \mathbb{P}\left(\frac{\prod^{\frac{1}{2} 4^{6}}}{5}\right)=S_{136} \circ S_{24} \circ A_{125} \circ A_{34} \\
= & {[1+(12)+(13)+(23)+(123)+(321)] } \\
& \circ[1+(24)] \\
& \circ[1-(12)-(13)-(23)+(123)+(321)] \\
& \circ[1-(34)]
\end{aligned}
$$

where $S_{136}$ denotes symmetrization between the 1 st, $3 \mathrm{rd}$, 6th index; similarly, $A_{34}$ denotes antisymmetrization between 


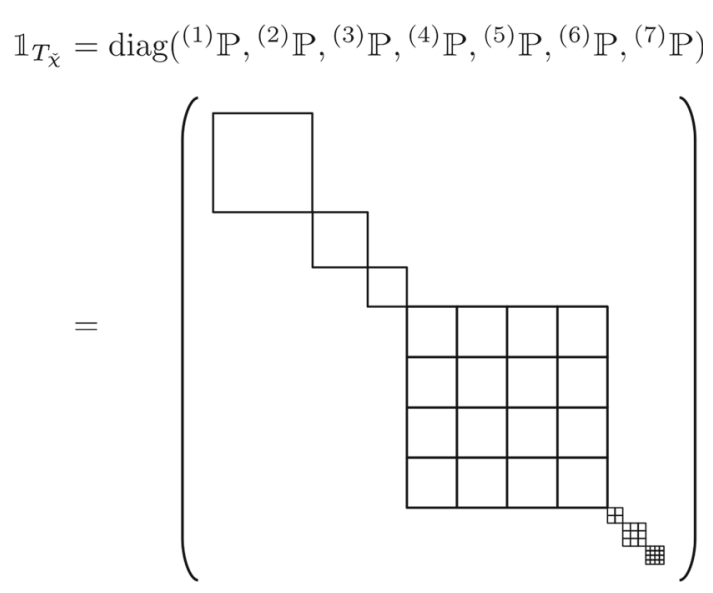

Fig. 2 The resolution of the identity on the 576-dimensional vector space $T_{\check{\chi}}$ has a block-diagonal structure in terms of the canonical Young projection operators ${ }^{\{I\}} \mathbb{P}: T_{\check{\chi}} \rightarrow T_{\check{\chi}}^{I}$ with $I=1, \ldots, 7$. We depict these operators by $1 \times 1$ blocks, with the relative width of $f^{\lambda_{I}} \times d_{I} / 576$. Provided $f^{\lambda_{I}}>1$, these operators can be further decomposed into noncanonical operators ${ }^{(I, j)} \mathbb{P}: T_{\check{\chi}}^{I} \rightarrow T_{\check{\chi}}^{I}$ with $j=1, \ldots, f^{\lambda_{I}}$. We depict this internal ambiguity by inserting $f^{\lambda_{I}} \times f^{\lambda_{I}}$ smaller blocks

the 3 rd and 4 th index, and so on. In the last lines we switched to the more convenient cycle notation. Note that in our conventions the Young symmetrizers are not normalized.

The exact expressions are lengthy, so we make some comments:

(i) Using the Young decomposition technique, the 576dimensional tensor space of $\check{\chi}_{\alpha \beta \gamma \mu \nu \rho}$, call it $T_{\check{\chi}}$, can be decomposed into the direct sum of lower-dimensional vector spaces $T_{\check{\chi}}^{I}$ with $I=1, \ldots, 7$. Denoting the projection operators onto these lower-dimensional subspaces via ${ }^{(I)} \mathbb{P}: T_{\check{\chi}} \rightarrow T_{\check{\chi}}^{I}$, the identity element on $T_{\check{\chi}}$ can be written as

$$
\mathbb{1}_{T_{\check{\chi}}}=\bigoplus_{I=1}^{7}{ }^{(I)} \mathbb{P} \quad \Rightarrow \quad{ }^{(I)} \check{\chi}:={ }^{(I)} \mathbb{P}(\check{\chi})
$$

(ii) In a suitable matrix representation of $T_{\check{\chi}}$, call it $\rho\left(T_{\check{\chi}}\right)=$ $\mathbb{R}^{576}$, the operators ${ }^{(I)} \mathbb{P}$ can be thought of as $\left(f^{\lambda_{I}} d_{I}\right) \times$ $\left(f^{\lambda_{I}} d_{I}\right)$ matrices, where $f^{\lambda_{I}}$ denotes the degeneracy of $T_{\check{\chi}}^{I}$.

(iii) On $T_{\check{\chi}}^{I}$, various auxiliary projection operators ${ }^{(I, j)} \mathbb{P}$ can be defined such that

$$
{ }^{(I)} \mathbb{P}=\bigoplus_{j=1}^{f^{\lambda}}{ }^{(I, j)} \mathbb{P} .
$$

We emphasize that the operators $(I, j) \mathbb{P}$ are not canonical, that is, they are only determined up to arbitrary linear transformations $G: \rho\left(T_{\check{\chi}}^{I}\right) \rightarrow \rho\left(T_{\check{\chi}}^{I}\right)$. For a graphical representation of statements (i)-(iii), see Fig. 2.
We now use computer algebra $[10,16]$ to obtain explicit expressions which are a bit lengthy. To compress our results, we define a projector $\mathbf{C}$ (its index representation $C_{\alpha \beta \gamma \delta}^{\rho \sigma \omega \lambda}$ ) by

$$
\begin{aligned}
\mathbf{C} & :=\frac{1}{4}[1-(12)-(45)+(12)(45)] \\
C_{\alpha \beta \gamma \delta}^{\rho \sigma \omega \lambda} & :=\frac{1}{4} \delta_{\alpha \beta}^{\rho \sigma} \delta_{\gamma \delta}^{\omega \lambda}, \quad \delta_{\alpha \beta}^{\rho \sigma}:=\delta_{\alpha}^{\rho} \delta_{\beta}^{\sigma}-\delta_{\alpha}^{\sigma} \delta_{\beta}^{\rho} .
\end{aligned}
$$

Observe that $\mathbf{C}^{2}=\mathbf{C}$, indeed. This projector antisymmetrizes in the two index pairs $\alpha \beta$ and $\gamma \delta$ such that any constitutive tensor satisfying the relation $\check{\chi}_{\alpha \beta \mu \gamma \delta v}=\check{\chi}_{[\alpha \beta] \mu[\gamma \delta] \nu}$ has eigenvalue 1 with respect to this operator:

$C_{\alpha \beta \gamma \delta}^{\rho \sigma \omega \lambda} \check{\chi}_{\rho \sigma \mu \omega \lambda \nu}=\check{\chi}_{\alpha \beta \mu \gamma \delta \nu}$

In general, $C_{\alpha \beta \gamma \delta}^{\rho \sigma \omega \lambda}$ projects a general order-6 tensor without any symmetries, call it $T_{\alpha \beta \mu \gamma \delta \nu}$, onto a tensor that has the symmetries of the constitutive tensor. In that sense we make use of this projector to shorten our exact expressions for the irreducible decomposition of the constitutive tensor by a factor of 4. We obtain:

$$
\begin{aligned}
& \text { (1) } \check{\chi}_{\alpha \beta \mu \gamma \delta v} \\
& =\frac{1}{20} C_{\alpha \beta \gamma \delta}^{\rho \sigma \omega \lambda}\left(-\check{\chi}_{\rho \sigma \omega \lambda \mu \nu}-\check{\chi}_{\rho \sigma \omega \lambda \nu \mu}+\check{\chi}_{\rho \sigma \mu \omega \lambda \nu}\right. \\
& +\check{\chi}_{\rho \sigma \mu \omega \nu \lambda}+\check{\chi}_{\rho \sigma v \omega \lambda \mu}+\check{\chi}_{\rho \sigma v \omega \mu \lambda}-\check{\chi}_{\rho \omega \sigma \lambda \mu \nu}-\check{\chi}_{\rho \omega \sigma \lambda \nu \mu} \\
& -\check{\chi}_{\rho \omega \lambda \sigma \mu \nu}-\check{x}_{\rho \omega \lambda \sigma \nu \mu}-\check{x}_{\rho \omega \mu \sigma \nu \lambda}-\check{\chi}_{\rho \omega \mu \lambda \nu \sigma} \\
& -\check{\chi}_{\rho \omega \nu \sigma \mu \lambda}-\check{\chi}_{\rho \omega \nu \lambda \mu \sigma}+\check{\chi}_{\rho \mu \sigma \omega \lambda \nu}+\check{\chi}_{\rho \mu \sigma \omega \nu \lambda} \\
& +\check{\chi}_{\rho \mu \omega \sigma \lambda \nu}-\check{\chi}_{\rho \mu \omega \lambda \nu \sigma}-\check{\chi}_{\rho \mu \nu \sigma \omega \lambda}+\check{\chi}_{\rho \mu \nu \omega \lambda \sigma}+\check{\chi}_{\rho \nu \sigma \omega \lambda \mu} \\
& +\check{\chi}_{\rho v \sigma \omega \mu \lambda}+\check{\chi}_{\rho v \omega \sigma \lambda \mu}-\check{\chi}_{\rho v \omega \lambda \mu \sigma}-\check{\chi}_{\rho v \mu \sigma \omega \lambda}+\check{\chi}_{\rho v \mu \omega \lambda \sigma} \\
& +\check{\chi}_{\omega \lambda \rho \sigma \mu \nu}+\check{\chi}_{\omega \lambda \rho \sigma \nu \mu}-\check{\chi}_{\omega \lambda \mu \rho \sigma \nu}-\check{\chi}_{\omega \lambda \mu \rho \nu \sigma}-\check{\chi}_{\omega \lambda \nu \rho \sigma \mu} \\
& -\check{\chi}_{\omega \lambda \nu \rho \mu \sigma}+\check{\chi}_{\omega \mu \rho \sigma \lambda \nu}+\check{\chi}_{\omega \mu \rho \sigma v \lambda}-\check{\chi}_{\omega \mu \lambda \rho \sigma \nu}-\check{\chi}_{\omega \mu \lambda \rho \nu \sigma} \\
& -\check{\chi}_{\omega \nu \mu \rho \sigma \lambda}-\check{\chi}_{\omega \mu \nu \rho \sigma \lambda}-\check{\chi}_{\omega \mu \nu \rho \lambda \sigma}+\check{\chi}_{\omega \nu \rho \sigma \lambda \mu}+\check{\chi}_{\omega \nu \rho \sigma \mu \lambda} \\
& \left.-\check{\chi}_{\omega \nu \lambda \rho \sigma \mu}-\check{\chi}_{\omega \nu \lambda \rho \mu \sigma}-\check{\chi}_{\omega \nu \mu \rho \lambda \sigma}\right), \\
& \text { (2) } \check{\chi}_{\alpha \beta \mu \gamma \delta \nu} \\
& =\frac{1}{18} C_{\alpha \beta \gamma \delta}^{\rho \sigma \omega \lambda}\left(-\check{\chi}_{\rho \sigma \omega \lambda \mu \nu}-\check{\chi}_{\rho \sigma \omega \lambda \nu \mu}+\check{\chi}_{\rho \sigma \mu \omega \lambda \nu}\right. \\
& +\check{\chi}_{\rho \sigma \mu \omega \nu \lambda}+\check{\chi}_{\rho \sigma \nu \omega \lambda \mu}+\check{\chi}_{\rho \sigma \nu \omega \mu \lambda}-\check{\chi}_{\rho \omega \sigma \lambda \mu \nu} \\
& -\check{\chi}_{\rho \omega \sigma \lambda \nu \mu}-\check{\chi}_{\rho \omega \lambda \sigma \mu \nu}-\check{\chi}_{\rho \omega \lambda \sigma v \mu}-\check{\chi}_{\rho \omega \mu \sigma \nu \lambda} \\
& -\check{\chi}_{\rho \omega \mu \lambda \nu \sigma}-\check{\chi}_{\rho \omega \nu \sigma \mu \lambda}-\check{\chi}_{\rho \omega \nu \lambda \mu \sigma}+\check{\chi}_{\rho \mu \sigma \omega \lambda \nu} \\
& +\check{\chi}_{\rho \mu \sigma \omega \nu \lambda}+\check{\chi}_{\rho \mu \omega \sigma \lambda \nu}-\check{\chi}_{\rho \mu \omega \lambda \nu \sigma}-\check{\chi}_{\rho \mu \nu \sigma \omega \lambda}+\check{\chi}_{\rho \mu \nu \omega \lambda \sigma} \\
& +\check{\chi}_{\rho v \sigma \omega \lambda \mu}+\check{\chi}_{\rho v \sigma \omega \mu \lambda}+\check{\chi}_{\rho \nu \omega \sigma \lambda \mu}-\check{\chi}_{\rho \nu \omega \lambda \mu \sigma}-\check{\chi}_{\rho \nu \mu \sigma \omega \lambda} \\
& +\check{\chi}_{\rho v \mu \omega \lambda \sigma}+\check{\chi}_{\omega \lambda \rho \sigma \mu \nu}+\check{\chi}_{\omega \lambda \rho \sigma v \mu}-\check{\chi}_{\omega \lambda \mu \rho \sigma \nu} \\
& -\check{\chi}_{\omega \lambda \mu \rho \nu \sigma}-\check{\chi}_{\omega \lambda \nu \rho \sigma \mu}-\check{\chi}_{\omega \lambda \nu \rho \mu \sigma}+\check{\chi}_{\omega \mu \rho \sigma \lambda \nu} \\
& +\check{\chi}_{\omega \mu \rho \sigma \nu \lambda}-\check{\chi}_{\omega \mu \lambda \rho \sigma \sigma}-\check{x}_{\omega \mu \lambda \rho \nu \sigma}-\check{\chi}_{\omega \nu \mu \rho \sigma \lambda}-\check{\chi}_{\omega \mu \nu \rho \sigma \lambda} \\
& -\check{\chi}_{\omega \mu \nu \rho \rho \sigma}+\check{\chi}_{\omega \nu \rho \sigma \lambda \mu}+\check{\chi}_{\omega \nu \rho \sigma \mu \lambda} \\
& \left.-\check{\chi}_{\omega \nu \lambda \rho \sigma \mu}-\check{\chi}_{\omega \nu \lambda \rho \mu \sigma}-\check{\chi}_{\omega \nu \mu \rho \lambda \sigma}\right), \\
& \text { (3) } \check{\chi}_{\alpha \beta \mu \gamma \delta \nu} \\
& =\frac{1}{36} C_{\alpha \beta \gamma \delta}^{\rho \sigma \omega \lambda}\left(-\check{\chi}_{\rho \sigma \omega \lambda \mu \nu}+\check{\chi}_{\rho \sigma \omega \lambda \nu \mu}+2 \check{\chi}_{\rho \sigma \omega \mu \nu \lambda}\right.
\end{aligned}
$$


$+\check{\chi}_{\rho \sigma \mu \omega \lambda \nu}+\check{\chi}_{\rho \sigma \mu \omega \nu \lambda}-\check{\chi}_{\rho \sigma \nu \omega \lambda \mu}-\check{\chi}_{\rho \sigma \nu \omega \mu \lambda}$

$-\check{\chi}_{\rho \omega \sigma \lambda \mu \nu}+\check{\chi}_{\rho \omega \sigma \lambda \nu \mu}+2 \check{\chi}_{\rho \omega \sigma \mu \nu \lambda}+\check{\chi}_{\rho \omega \lambda \sigma \mu \nu}$

$-\check{\chi}_{\rho \omega \lambda \sigma \nu \mu}-2 \check{\chi}_{\rho \omega \lambda \mu \nu \sigma}+2 \check{\chi}_{\rho \omega \mu \sigma \lambda \nu}+\check{\chi}_{\rho \omega \mu \sigma \nu \lambda}$

$-\check{\chi}_{\rho \omega \mu \lambda \nu \sigma}-2 \check{\chi}_{\rho \omega \nu \sigma \lambda \mu}-\check{\chi}_{\rho \omega \nu \sigma \mu \lambda}+\check{\chi}_{\rho \omega \nu \lambda \mu \sigma}$

$+\check{\chi}_{\rho \mu \sigma \omega \lambda \nu}+\check{\chi}_{\rho \mu \sigma \omega \nu \lambda}+\check{\chi}_{\rho \mu \omega \sigma \lambda \nu}+2 \check{\chi}_{\rho \mu \omega \sigma \nu \lambda}$

$+\check{\chi}_{\rho \mu \omega \lambda \nu \sigma}+\check{\chi}_{\rho \mu \nu \sigma \omega \lambda}-\check{\chi}_{\rho \mu \nu \omega \lambda \sigma}-\check{\chi}_{\rho \nu \sigma \omega \lambda \mu}$

$-\check{\chi}_{\rho \nu \sigma \omega \mu \lambda}-\check{x}_{\rho \nu \omega \sigma \lambda \mu}-2 \check{\chi}_{\rho \nu \omega \sigma \mu \lambda}-\check{x}_{\rho \nu \omega \lambda \mu \sigma}$

$-\check{\chi}_{\rho v \mu \sigma \omega \lambda}+\check{\chi}_{\rho \nu \mu \omega \lambda \sigma}-\check{\chi}_{\omega \lambda \rho \sigma \mu \nu}+\check{\chi}_{\omega \lambda \rho \sigma v \mu}$

$+2 \check{\chi}_{\omega \lambda \rho \mu \nu \sigma}+\check{\chi}_{\omega \lambda \mu \rho \sigma \nu}+\check{\chi}_{\omega \lambda \mu \rho \nu \sigma}-\check{\chi}_{\omega \lambda \nu \rho \sigma \mu}$

$-\check{\chi}_{\omega \lambda \nu \rho \mu \sigma}-\check{\chi}_{\omega \mu \rho \sigma \lambda \nu}+\check{\chi}_{\omega \mu \rho \sigma \nu \lambda}+2 \check{\chi}_{\omega \mu \rho \lambda \nu \sigma}$

$+\check{\chi}_{\omega \mu \lambda \rho \sigma \nu}+\check{\chi}_{\omega \mu \lambda \rho \nu \sigma}-\check{\chi}_{\omega \mu \nu \rho \sigma \lambda}-\check{\chi}_{\omega \mu \nu \nu \rho \lambda \sigma}$

$+\check{\chi}_{\omega \nu \rho \sigma \lambda \mu}-\check{\chi}_{\omega v \rho \sigma \mu \lambda}-2 \check{\chi}_{\omega \nu \rho \lambda \mu \sigma}-\check{\chi}_{\omega \nu \lambda \rho \rho \sigma \mu}$

$-\check{\chi}_{\omega \nu \lambda \rho \mu \sigma}+\check{\chi}_{\omega \nu \mu \rho \sigma \lambda}+\check{\chi}_{\omega \nu \mu \rho \lambda \sigma}$

$\left.-2 \check{\chi}_{\mu \nu \rho \sigma \omega \lambda}+2 \check{\chi}_{\mu \nu \rho \omega \lambda \sigma}+2 \check{\chi}_{\mu \nu \omega \rho \sigma \lambda}+2 \check{\chi}_{\mu \nu \omega \rho \lambda \sigma)}\right)$,

(4) $\check{\chi}_{\alpha \beta \mu \gamma \delta \nu}$

$$
\begin{aligned}
& =\frac{4}{45} C_{\alpha \beta \gamma \delta}^{\rho \sigma \omega \lambda}\left(-\check{\chi}_{\rho \sigma \omega \lambda \mu \nu}+\check{\chi}_{\rho \sigma \omega \lambda \nu \mu}+4 \check{\chi}_{\rho \sigma \mu \omega \lambda \nu}\right. \\
& +\check{\chi}_{\rho \sigma \mu \omega \nu \lambda}-\check{\chi}_{\rho \sigma \nu \omega \mu \lambda}+\check{\chi}_{\rho \omega \sigma \lambda \mu \nu}+2 \check{\chi}_{\rho \omega \sigma \lambda \nu \mu} \\
& -\check{\chi}_{\rho \omega \sigma \mu \nu \lambda}+\check{\chi}_{\rho \omega \lambda \sigma \mu \nu}+\check{\chi}_{\rho \omega \lambda \sigma \nu \mu}+\check{\chi}_{\rho \omega \lambda \mu \nu \sigma} \\
& +4 \check{\chi}_{\rho \omega \mu \sigma \lambda \nu}+\check{\chi}_{\rho \omega \mu \sigma \nu \lambda}+\check{\chi}_{\rho \omega \mu \lambda \nu \sigma}+\check{\chi}_{\rho \omega \nu \sigma \mu \lambda} \\
& +2 \check{\chi}_{\rho \omega \nu \lambda \mu \sigma}+\check{\chi}_{\rho \mu \sigma \omega \lambda \nu}-2 \check{\chi}_{\rho \mu \sigma \omega \nu \lambda}-\check{\chi}_{\rho \mu \omega \sigma \lambda \nu} \\
& -\check{x}_{\rho \mu \omega \sigma \nu \lambda}+2 \check{x}_{\rho \mu \omega \lambda \nu \sigma}+2 \check{x}_{\rho \mu \nu \sigma \omega \lambda}-\check{x}_{\rho \mu \nu \omega \lambda \sigma} \\
& -\check{\chi}_{\rho \nu \sigma \omega \lambda \mu}-2 \check{x}_{\rho \nu \sigma \omega \mu \lambda}-2 \check{x}_{\rho \nu \omega \sigma \lambda \mu}+\check{x}_{\rho \nu \omega \sigma \mu \lambda} \\
& +2 \check{x}_{\rho \nu \omega \lambda \mu \sigma}+\check{x}_{\rho \nu \mu \sigma \omega \lambda}+\check{x}_{\rho \nu \mu \omega \lambda \sigma}+\check{x}_{\omega \lambda \rho \sigma \mu \nu} \\
& -2 \check{\chi}_{\omega \lambda \rho \mu \nu \nu \sigma}-\check{x}_{\omega \lambda \mu \rho \nu \sigma}-\check{x}_{\omega \mu \rho \sigma \lambda \nu}-\check{\chi}_{\omega \mu \rho \lambda \nu \sigma \sigma} \\
& -\check{\chi}_{\omega \mu \lambda \rho \sigma \sigma \nu}-2 \check{\chi}_{\omega \mu \lambda \rho \nu \sigma}+\check{\chi}_{\omega \mu \nu \rho \lambda \sigma}-\check{\chi}_{\omega \nu \rho \sigma \lambda \mu} \\
& +2 \check{\chi}_{\omega \nu \rho \sigma \mu \lambda}+\check{\chi}_{\omega \nu \rho \lambda \mu \sigma-}-\check{\chi}_{\omega \nu \mu \rho \sigma \lambda} \\
& \left.+\check{\chi}_{\omega \nu \mu \rho \lambda \sigma}+\check{\chi}_{\mu \nu \rho \sigma \omega \lambda}-2 \check{\chi}_{\mu \nu \omega \rho \sigma \lambda}-\check{\chi}_{\mu \nu \omega \rho \lambda \sigma}\right),
\end{aligned}
$$

(5) $\check{\chi}_{\alpha \beta \mu \gamma \delta \nu}$

$$
=\frac{1}{36} C_{\alpha \beta \gamma \delta}^{\rho \sigma \omega \lambda}\left(\check{\chi}_{\rho \sigma \omega \lambda \mu \nu}+\check{\chi}_{\rho \sigma \omega \lambda \nu \mu}-2 \check{\chi}_{\rho \sigma \omega \mu \nu \lambda}\right.
$$

$+2 \check{\chi}_{\rho \sigma \mu \omega \lambda \nu}-\check{\chi}_{\rho \sigma \mu \omega \nu \lambda}-\check{\chi}_{\rho \sigma \nu \omega \mu \lambda}+3 \check{\chi}_{\rho \omega \sigma \lambda \mu \nu}$

$-\check{\chi}_{\rho \omega \sigma \lambda \nu \mu}+\check{\chi}_{\rho \omega \lambda \sigma \mu \nu}-3 \check{\chi}_{\rho \omega \lambda \sigma \nu \mu}+2 \check{\chi}_{\rho} \rho \omega \mu \lambda \nu$

$+\check{\chi} \rho \omega \mu \sigma \nu \lambda+3 \check{\chi}_{\rho \omega \mu \lambda \nu \sigma}+2 \check{\chi}_{\rho \omega \nu \sigma \lambda \mu}-3 \check{\chi}_{\rho \omega \nu \sigma \mu \lambda}$

$-\check{\chi}_{\rho \omega \nu \lambda \mu \sigma}-\check{x}_{\rho \mu \sigma \omega \lambda \nu}+5 \check{\chi}_{\rho \mu \sigma \omega \nu \lambda}-3 \check{x}_{\rho \mu \omega \sigma \lambda \nu}$

$-\check{\chi}_{\rho \mu \omega \lambda \nu \sigma}-\check{\chi}_{\rho \mu \nu \sigma \omega \lambda}-\check{\chi}_{\rho \mu \nu \omega \lambda \sigma}-\check{\chi}_{\rho \nu \sigma \omega \lambda \mu}$

$+\check{\chi}_{\rho \nu \sigma \omega \mu \lambda}+\check{\chi}_{\rho v \omega \sigma \lambda \mu}-5 \check{\chi}_{\rho \nu \omega \lambda \mu \sigma}+3 \check{\chi}_{\rho \nu \mu \sigma \omega \lambda}$

$-\check{\chi}_{\rho \nu \mu \omega \lambda \sigma}+\check{\chi}_{\omega \lambda \rho \sigma \mu \nu}+\check{\chi}_{\omega \lambda \rho \sigma \nu \mu}+2 \check{\chi}_{\omega \lambda \rho \mu \nu \sigma}$

$-\check{\chi}_{\omega \lambda \mu \rho \nu \sigma}+2 \check{\chi}_{\omega \lambda \nu \rho \sigma \mu}-\check{\chi}_{\omega \lambda \nu \rho \mu \sigma}-\check{\chi}_{\omega \mu \rho \sigma \lambda \nu}$

$-5 \check{\chi}_{\omega \mu \rho \sigma \nu \lambda}-\check{x}_{\omega \mu \lambda \rho \sigma \nu}+\check{\chi}_{\omega \mu \lambda \rho \nu \sigma}-\check{x}_{\omega \mu \nu \rho \sigma \alpha}$

$-3 \check{\chi}_{\omega \mu \nu \rho \lambda \sigma}+3 \check{\chi}_{\omega \nu \rho \sigma \lambda \mu}-\check{\chi}_{\omega \nu \rho \sigma \mu \lambda}-\check{\chi}_{\omega \nu \lambda \rho \sigma \sigma \mu}$

$+5 \check{\chi}_{\omega \nu \lambda \rho \mu \sigma}-\check{\chi}_{\omega \nu \mu \rho \sigma \lambda}+\check{\chi}_{\omega \nu \mu \rho \lambda \sigma}$

$-2 \check{\chi}_{\mu \nu \rho \omega \lambda \sigma}+2 \check{\chi}_{\mu \nu \omega \rho \sigma \lambda)}$,

(6) $\check{\chi}_{\alpha \beta \mu \gamma \delta \nu}$

$$
\begin{aligned}
= & \frac{1}{18} C_{\alpha \beta \gamma \delta}^{\rho \sigma \omega \lambda}\left(-2 \check{\chi}_{\rho \sigma \omega \mu \nu \lambda}+3 \check{\chi}_{\rho \sigma \mu \omega \lambda \nu}+\check{\chi}_{\rho \sigma \nu \omega \lambda \mu}\right. \\
& -\check{x}_{\rho \omega \sigma \lambda \mu \nu}+\check{\chi}_{\rho \omega \sigma \lambda \nu \mu}-3 \check{x}_{\rho \omega \lambda \sigma \mu \nu}-\check{x}_{\rho \omega \lambda \sigma \nu \mu} \\
& -4 \check{\chi}_{\rho \omega \mu \sigma \lambda \nu}-3 \check{x}_{\rho \omega \mu \sigma \nu \lambda}-\check{x}_{\rho \omega \mu \lambda \nu \sigma}-4 \check{x}_{\rho \omega \nu \sigma \lambda \mu}
\end{aligned}
$$

$$
\begin{aligned}
& -\check{\chi}_{\rho \omega \nu \sigma \mu \lambda}+\check{\chi}_{\rho \omega \nu \lambda \mu \sigma}-3 \check{\chi}_{\rho \mu \sigma \omega \nu \lambda}+\check{\chi}_{\rho \mu \omega \sigma \lambda \nu} \\
& +\check{\chi}_{\rho \mu \omega \lambda \nu \sigma}+\check{\chi}_{\rho \mu \nu \sigma \omega \lambda}-\check{\chi}_{\rho \nu \sigma \omega \mu \lambda}-\check{\chi}_{\rho \nu \omega \sigma \lambda \mu} \\
& +3 \check{\chi}_{\rho \nu \omega \lambda \mu \sigma}-\check{\chi}_{\rho \nu \mu \sigma \omega \lambda}-\check{\chi}_{\omega \lambda \rho \sigma \mu \nu \nu}-\check{\chi}_{\omega \lambda \rho \sigma \sigma \nu \mu} \\
& +2 \check{\chi}_{\omega \lambda \rho \mu \nu \sigma}-\check{\chi}_{\omega \lambda \mu \rho \sigma \nu}+\check{\chi}_{\omega \lambda \mu \rho \nu \sigma}+\check{\chi}_{\omega \lambda \nu \rho \sigma \mu} \\
& +\check{\chi}_{\omega \lambda \nu \rho \mu \sigma \sigma}+3 \check{\chi}_{\omega \mu \rho \sigma \lambda \nu}-\check{\chi}_{\omega \mu \rho \sigma \nu \lambda}+\check{\chi}_{\omega \mu \lambda \rho \sigma \nu} \\
& +3 \check{\chi}_{\omega \mu \lambda \rho \nu \sigma}+\check{\chi}_{\omega \mu \nu \rho \sigma \lambda}-\check{x}_{\omega \mu \nu \rho \lambda \sigma}+\check{\chi}_{\omega \nu \rho \sigma \lambda \mu} \\
& -3 \check{\chi}_{\omega v \rho \sigma \mu \lambda}+\check{\chi}_{\omega \nu \lambda \rho \sigma \mu}+\check{\chi}_{\omega \nu \lambda \rho \mu \sigma}+\check{\chi}_{\omega \nu \mu \rho \sigma \lambda} \\
& \left.-3 \check{\chi}_{\omega \nu \mu \rho \lambda \sigma}-2 \check{\chi}_{\mu \nu \rho \omega \lambda \sigma}+2 \check{\chi}_{\mu \nu \omega \rho \sigma \lambda}\right) \text {, }
\end{aligned}
$$

(7) $\check{\chi}$

$$
\begin{aligned}
& =\frac{1}{20} C_{\alpha \beta \gamma \delta}^{\rho \sigma \omega \lambda}\left(2 \check{\chi}_{\rho \sigma \omega \lambda \mu \nu}+2 \check{\chi}_{\rho \sigma \omega \mu \nu \lambda}+4 \check{\chi}_{\rho \sigma \mu \omega \lambda \nu}\right. \\
& -2 \check{\chi}_{\rho \sigma \mu \omega \nu \lambda}-2 \check{\chi}_{\rho \sigma \nu \omega \lambda \mu}+3 \check{\chi}_{\rho \omega \sigma \lambda \mu \nu}-3 \check{\chi}_{\rho \omega \sigma \lambda \nu \mu} \\
& -\check{\chi}_{\rho \omega \lambda \sigma \mu \nu}+\check{\chi}_{\rho \omega \lambda \sigma \nu \mu}-2 \check{\chi}_{\rho \omega \mu \sigma \lambda \nu}-\check{\chi}_{\rho \omega \mu \sigma \nu \lambda} \\
& +3 \check{\chi}_{\rho \omega \mu \lambda \nu \sigma}+2 \check{\chi}_{\rho \omega \nu \sigma \lambda \mu}+\check{\chi}_{\rho \omega \nu \sigma \mu \lambda}-3 \check{\chi}_{\rho \omega \nu \lambda \mu \sigma} \\
& -2 \check{\chi}_{\rho \mu \sigma \omega \lambda \nu}+\check{\chi}_{\rho \mu \sigma \omega \nu \lambda}-3 \check{\chi}_{\rho \mu \omega \sigma \lambda \nu}-3 \check{\chi}_{\rho \mu \omega \lambda \nu \sigma} \\
& -3 \check{\chi}_{\rho \mu \nu \sigma \omega \lambda}+3 \check{\chi}_{\rho \nu \sigma \omega \mu \lambda}+3 \check{\chi}_{\rho \nu \omega \sigma \lambda \mu}-\check{\chi}_{\rho \nu \omega \lambda \mu \sigma} \\
& +3 \check{\chi}_{\rho \nu \mu \sigma \omega \lambda}-2 \check{\chi}_{\rho \nu \mu \omega \lambda \sigma}-\check{x}_{\omega \lambda \rho \sigma \mu \nu}-\check{\chi}_{\omega \lambda \rho \sigma \nu \mu} \\
& +\check{\chi}_{\omega \lambda \mu \rho \nu \sigma}-2 \check{\chi}_{\omega \lambda \nu \rho \sigma \mu}+\check{\chi}_{\omega \lambda \nu \rho \mu \sigma}+\check{\chi}_{\omega \mu \rho \sigma \lambda v} \\
& +5 \check{\chi}_{\omega \mu \rho \sigma \nu \lambda}+\check{\chi}_{\omega \mu \lambda \rho \sigma \nu}+\check{\chi}_{\omega \mu \lambda \rho \nu \sigma}+\check{\chi}_{\omega \mu \nu \rho \sigma \lambda} \\
& +\check{\chi}_{\omega \mu \nu \rho \lambda \sigma}-\check{\chi}_{\omega \nu \rho \sigma \lambda \mu}-\check{\chi}_{\omega \nu \rho \sigma \mu \lambda}+\check{\chi}_{\omega \nu \lambda \rho \sigma \mu} \\
& \left.-5 \check{\chi}_{\omega \nu \lambda \rho \mu \sigma}+\check{\chi}_{\omega \nu \mu \rho \sigma \lambda}-\check{\chi}_{\omega \nu \mu \rho \lambda \sigma}+2 \check{\chi}_{\mu \nu \rho \omega \lambda \sigma}\right) \text {. }
\end{aligned}
$$

It has been verified by computer algebra that the above expressions indeed belong to orthogonal subspaces.

\section{References}

1. R. Aldrovandi, J.G. Pereira, Teleparallel Gravity: An Introduction (Springer, Dordrecht, 2013)

2. P. Baekler, A. Favaro, Y. Itin, F.W. Hehl, The Kummer tensor density in electrodynamics and in gravity. Ann. Phys. (NY) 349, 297324 (2014). arXiv:1403.3467

3. P. Baekler, F.W. Hehl, Beyond Einstein-Cartan gravity: quadratic torsion and curvature invariants with even and odd parity including all boundary terms. Class. Quantum Gravity 28, 215017 (2011). arXiv: 1105.3504

4. P. Baekler, F.W. Hehl, J.M. Nester, Poincaré gauge theory of gravity: Friedman cosmology with even and odd parity modes. Analytic Part. Phys. Rev. D 83, 024001 (2011). arXiv:1009.5112

5. A. O. Barut, R. Raczka, Theory of Group Representations and Applications (PWN, Warszawa, 1977, and World Scientific, Singapore, 1986)

6. M. Blagojević, B. Cvetković, Y.N. Obukhov, Generalized plane waves in Poincaré gauge theory of gravity. Phys. Rev. D 96, 064031 (2017). arXiv: 1708.08766

7. M. Blagojević, B. Cvetković, General Poincaré gauge theory: Hamiltonian structure and particle spectrum. Phys. Rev. D 98, 024014 (2018). arXiv: 1804.05556

8. M. Blagojević, F.W. Hehl (eds.), Gauge Theories of Gravitation: A Reader with Commentaries (Imperial College Press, London, 2013). See arXiv: 1210.3775

9. C.G. Böhmer, R.J. Downes, From continuum mechanics to general relativity. Int. J. Mod. Phys. D 23, 1442015 (2014). arXiv: 1405.4728 
10. J. Boos, Irreducible decomposition of the rank- 6 constitutive tensor (online computer algebra code). see http://spintwo.net/static/2018. 07.17/. Last retrieved: 17 Jul 2018

11. Y.M. Cho, Einstein Lagrangian as the translational Yang-Mills Lagrangian. Phys. Rev. D 14, 2521 (1976)

12. D.H. Delphenich, The use of the teleparallelism connection in continuum mechanics. Math. Mech. Solids 21, 1260-1275 (2016). arXiv: 1305.3477

13. A. Einstein, The Meaning of Relativity, 5th edn. (Princeton University Press, Princeton, 1955)

14. R. Ferraro, M.J. Guzmán, Hamiltonian formulation of teleparallel gravity. Phys. Rev. D 94, 104045 (2016). arXiv:1609.06766

15. M. Hamermesh, Group Theory and Its Applications to Physical Problems (Addison-Wesley, Reading, 1962)

16. A. C. Hearn, REDUCE User's Manual, Version 3.5, RAND Publication CP78 (Rev. 10/93). The RAND Corporation, Santa Monica, CA 90407-2138, USA (1993). Nowadays Reduce is freely available for download; for details see [http://reduce-algebra.com] and [http://sourceforge.net]

17. F.W. Hehl, Four lectures on Poincaré gauge field theory, in: Proceedings of the 6th Course of the School of Cosmology and Gravitation on Spin, Torsion, Rotation, and Supergravity, held at Erice, Italy, May 1979, P.G. Bergmann, V. de Sabbata, eds. (Plenum, New York 1980) pp. 5-61; see also the author's homepage: http://www. thp.uni-koeln.de/gravitation/mitarbeiter/hehl.html

18. F.W. Hehl, Y. Itin, Y.N. Obukhov, On Kottler's path: origin and evolution of the premetric program in gravity and in electrodynamics. arXiv:1607.06159. A condensed version appeared in the Int. J. Mod. Phys. D 25, 1640016 (2016)

19. F.W. Hehl, B. Mashhoon, Nonlocal gravity simulates dark matter. Phys. Lett. B 673, 279 (2009). arXiv:0812.1059

20. F.W. Hehl, B. Mashhoon, A formal framework for a nonlocal generalization of Einstein's theory of gravitation. Phys. Rev. D 79, 064028 (2009). arXiv:0902.0560

21. F.W. Hehl, J. Nitsch, P. von der Heyde, Gravitation and the Poincaré gauge field theory with quadratic Lagrangian, in General Relativity and Gravitation: One Hundred Years After the Birth of Albert Einstein, vol. 1, ed. by A. Held (Plenum Press, New York, 1980), pp. 329-355

22. F.W. Hehl, Yu.N. Obukhov, Foundations of Classical Electrodynamics: Charge, Flux, and Metric (Birkhäuser, Boston, 2003)

23. F.W. Hehl, E. Tonti, Tonti diagrams for the teleparallelism theory of gravity (TG) and for the Poincaré gauge theory (PG), Verhandlungen DPG (German Physical Society) (VI) 52, 3/p.56 (2017)

24. P. von der Heyde, Is gravitation mediated by the torsion of spacetime? Z. Naturforsch. 31a, 1725-1726 (1976)

25. F.H. Ho, H. Chen, J.M. Nester, H.J. Yo, General Poincaré gauge theory cosmology. Chin. J. Phys. 53, 110109 (2015). arXiv: 1512.01202

26. M. Hohmann, L. Järv, M. Krššák, C. Pfeifer, Teleparallel theories of gravity as analogue of non-linear electrodynamics. Phys. Rev. D 97, 104042 (2018). arXiv: 1711.09930

27. M. Hohmann, M. Krššák, C. Pfeifer, U. Ualikhanova, Propagation of gravitational waves in teleparallel gravity theories. To be published (2018). arXiv:1807.04580

28. Y. Itin, Weak field reduction in teleparallel coframe gravity: vacuum case. J. Math. Phys. 46, 012501 (2005). arXiv:gr-qc/0409021

29. Y. Itin, On light propagation in premetric electrodynamics: the covariant dispersion relation. J. Phys. A 42, 475402 (2009). arXiv:0903.5520

30. Y. Itin, Skewon modification of the light cone structure. Phys. Rev. D 91, 085002 (2015). arXiv: 1407.6722

31. Y. Itin, F.W. Hehl, Y.N. Obukhov, Premetric equivalent of general relativity: teleparallelism. Phys. Rev. D 95, 084020 (2017). arXiv: 1611.05759
32. G.K. Karananas, The particle spectrum of parity-violating Poincaré gravitational theory. Class. Quantum Gravity 32, 055012 (2015). arXiv:1411.5613; corrigendum: ibid. 32, 089501 (2015)

33. V.A. Kostelecký, M. Mewes, Lorentz and diffeomorphism violations in linearized gravity. Phys. Lett. B 779, 136-142 (2018). arXiv: 1712.10268

34. R. Kubo (in cooperation with H. Ichimura, T. Usui, N. Hashitsume), Thermodynamics, 2nd printing (North Holland, Amsterdam, 1976)

35. J.W. Maluf, Dirac spinor fields in the teleparallel gravity: comment on 'Metric affine approach to teleparallel gravity'. Phys. Rev. D 67, 108501 (2003). arXiv:gr-qc/0304005

36. J.W. Maluf, The teleparallel equivalent of general relativity. Ann. Phys. (Berlin) 525, 339 (2013). arXiv:1303.3897

37. B. Mashhoon, Nonlocal Gravity (Oxford University Press, Oxford, 2017)

38. H. Meyer, Møller's tetrad theory of gravitation as a special case of a Poincaré gauge theory-a coincidence? Gen. Relativ. Gravit. 14, 531-547 (1982)

39. U. Muench, F. Gronwald, F.W. Hehl, A Small guide to variations in teleparallel gauge theories of gravity and the Kaniel-Itin model. Gen. Relativ. Gravit. 30, 933-961 (1998). arXiv:gr-qc/9801036

40. F. Müller-Hoissen, J. Nitsch, Teleparallelism—a viable theory of gravity? Phys. Rev. D 28, 718-728 (1983)

41. H.T. Nieh, M.L. Yan, An identity in Riemann-Cartan geometry. J. Math. Phys. 23, 373 (1982)

42. H.T. Nieh, A torsional topological invariant. Int. J. Mod. Phys. A 22, 5237-5244 (2007)

43. Y.N. Obukhov, Poincaré gauge gravity: selected topics. Int. J. Geom. Meth. Mod. Phys. 3, 95 (2006). arXiv:gr-qc/0601090

44. Y.N. Obukhov, Gravitational waves in Poincaré gauge gravity theory. Phys. Rev. D 95, 084028 (2017). arXiv:1702.05185

45. Y.N. Obukhov, Poincaré gauge gravity: an overview, Lecture in Tartu, Estonia (2017). Int. J. Geom. Methods Mod. Phys. 15, 1840005 (2018). arXiv:1805.07385

46. Y.N. Obukhov, E.W. Mielke, J. Budczies, F.W. Hehl, On the chiral anomaly in non-Riemannian spacetimes. Found. Phys. 27, 12211236 (1997). arXiv:gr-qc/9702011

47. Y.N. Obukhov, J.G. Pereira, Lessons of spin and torsion: Reply to 'Consistent coupling to Dirac fields in teleparallelism'. Phys. Rev. D 69, 128502 (2004). arXiv:gr-qc/0406015

48. C. Pellegrini, J. Plebanski, Tetrad fields and gravitational fields. Mat. Fys. Skr. Dan. Vid. Selsk. 2(4), 1-39 (1963)

49. M. Planck, Treatise on Thermodynamics, 3rd edition 1926 [translated from the 7th German edition (Dover, New York, 1945)]

50. E.J. Post, Formal Structure of Electromagnetics-General Covariance and Electromagnetics (North Holland, Amsterdam, 1962 and Dover, Mineola, 1997)

51. I. Prigogine, Thermodynamics of Irreversible Processes, 2 nd rev. ed. (Interscience, New York, 1965)

52. R.J. Rivers, Lagrangian theory for neutral massive spin-2 fields. Nuovo Cim. 34, 386-403 (1964)

53. J.A. Schouten, Ricci Calculus, 2nd edn. (Springer, Berlin, 1954)

54. J.J. Sławianowski, V. Kovalchuk, B. Gołubowska, A. Martens, E.E. Rożko, Space-time as a structured relativistic continuum. Math. Methods Appl. Sci. 41, 5404-5422 (2018)

55. R. Stanley, Hooks and Contents. Talk at Penn. State, 05 Dec 2008. http://www-math.mit.edu/ rstan/transparencies/hooks.pdf

56. E. Tonti, The Mathematical Structure of Classical and Relativistic Physics, A General Classification Diagram (Birkhäuser-Springer, New York, 2013)

57. H.-J. Treder (editor, with contributions by H.H.V. Borzeszkowski, U. Kasper, E. Kreisel, D.-E. Liebscher, H.-J. Treder), Gravitationstheorie und Äquivalenzprinzip (in German) (Akademie-Verlag, Berlin, 1971) 